\title{
La pobreza y el ciclo económico en México, 2005-2012*
}

\author{
Enrique Hernández Laos** y Araceli Benítez Lino***
}

\section{RESUMEN}

En el contexto de la gran recesión de 2008-2009, se analiza la trayectoria trimestral seguida por las tasas de pobreza alimentaria, estimadas a partir del Índice de Tendencia Laboral de la Pobreza del Consejo Nacional de Evaluación de la Política de Desarrollo Social (CONEVAL), y se examina su relación con el desenvolvimiento del mercado laboral mexicano entre 2005 y 2012. Las pruebas estadísticas aportadas indican que los efectos cuantitativos del desempleo abierto sobre la pobreza alimentaria son similares a los del aumento de la informalidad, la cual repercute con un rezago trimestral después de que se presenta. Se muestra, por último, cómo los efectos sectoriales y regionales del ciclo sobre el mercado de trabajo dieron lugar a una trayectoria convergente de las tasas de pobreza alimentaria de las entidades federativas, durante la fase recesiva del ciclo seguido por la economía mexicana.

Palabras clave: pobreza alimentaria, mercado laboral, desempleo, ciclo económico. Clasificación JEL: I32, E24, E30.

\section{Abstract}

The years 2008-2009 witnessed the worst financial crisis since the Great Depression. It is in this context that we discuss the quarterly trajectory of the index of extreme poverty in Mexico, based on estimates of the Consejo Nacional de Evaluación de la Política de Desarrollo Social (CONEVAL) (Mexican council for the evaluation of social development policies). The article examines the relation between this course and developments in Mexico's labour market between 2005 and 2012. Statistical data show that the quantitative effects of open unemployment rates on the index of extreme poverty are similar to those exerted by the growth of the informal economy, the impact of which is characterized by a quarterly lag. The essay shows how the sectorial and regional effects of the economic cycle on the labour market gave rise to a convergent path among the rates of extreme poverty in different states during the recessive phase of the cycle that affected the country's economy.

Keywords: extreme poverty, labor market, unemployment, business cycle. JEL classification: I32, E24, E30.

* Fecha de recepción: 18/09/2013. Fecha de aceptación: 07/03/2014. Los autores agradecen los comentarios y observaciones del doctor Fernando Cortés, del Centro de Estudios Sociológicos de El Colegio de México, a una versión anterior del presente escrito. Así mismo, agradecen los valiosos comentarios y sugerencias aportados por dos lectores anónimos.

** Profesor-investigador del Doctorado en Ciencias Económicas y del Programa de Posgrado en Estudios Sociales de la Universidad Autónoma Metropolitana. Correo electrónico: ehlaos@ prodigy.net.mx.

*** Egresada de la Maestría en Estudios Sociales, de la Línea de Economía Social de la Universidad Autónoma Metropolitana-Iztapalapa. Correo electrónico: aralin_1405@yahoo.com.mx. 


\section{INTRODUCCIÓN}

En la primera edición de sus Principios de economía, Alfred Marshall (1977, p. 591) señalaba que el aumento de la tasa de desempleo era consecuencia de la disminución de la actividad económica en la Inglaterra de finales del siglo XIX, y en referencia a la crisis de 1907, Beatrice Webb (citada por Nasar, 2011, p. 107) apuntaba, a su vez, que el incremento del desempleo era la causa principal del crecimiento de la pobreza. Desde entonces quedó establecida la relación entre el ciclo económico, el desempleo y la pobreza, lo que posteriormente se confirmó de manera dramática con la Gran Depresión de los años treinta y se volvió a ratificar recientemente con la profunda recesión de 2008-2009.

El estudio sistemático de esa relación comenzó hacia finales del siglo xx, con el análisis de la economía estadounidense. En México, el interés surgió con el examen de los efectos de la crisis de los setenta y cobró relevancia con las recesiones de los ochenta y de los noventa, al evidenciarse el severo empobrecimiento de la población mexicana conforme el colapso de la actividad económica acrecentaba el desempleo y los índices de informalidad. Ya en el nuevo siglo, las recesiones de principio y fin de la década pasada ratificaron la vigencia de la relación apuntada un siglo atrás por Marshall y Webb.

Las dos preguntas que tratamos de responder en este texto son las siguientes: ¿Cual es la relación que se observa entre la evolución del ciclo económico registrado en México de 2005 a 2012 y los cambios en la tasa de pobreza alimentaria (TPA)? ¿Qué papel habría jugado en esta relación el desempeño del mercado laboral mexicano? Para ello, analizamos el comportamiento de este mercado, tanto mediante la tasa de desempleo abierto (TDA) como de la tasa de ocupación en el sector informal (TOSI), durante el ciclo económico de 2005 a 2012 y tratamos de identificar sus efectos sobre la TPA en nuestro país. Con el fin de llevar a cabo este análisis utilizamos, con las adaptaciones que más adelante se explican, las estimaciones trimestrales del Índice de Tendencia Laboral de la Pobreza (ITLP), generadas por el Consejo Nacional de Evaluación de la Política de Desarrollo Social (CONEVAL), tanto a escala nacional como regional, en el periodo referido.

Los análisis aquí presentados ratifican, de manera relativamente clara, la estrecha relación que guarda el desempeño del mercado laboral en las diversas fases del ciclo económico con el notable incremento del desempleo, la informalidad y la pobreza en la etapa recesiva de 2008-2009, tendencia que se alargó, aunque menos dinámicamente, hasta la recuperación de la economía a partir de 2010. 
Los ejercicios estadísticos presentados, consistentes en análisis de regresión de datos panel, ponen de manifiesto que los efectos recesivos sobre el mercado laboral se transmitieron de manera diferencial, a través de los sectores económicos, hacia las regiones y entidades federativas del país, incidiendo con intensidad desigual sobre las tasas de pobreza alimentaria de los hogares mexicanos.

Por su origen externo, la gran recesión de fines de la década pasada inicialmente repercutió en el sector manufacturero exportador de la economía y posteriormente en los sectores orientados al mercado interno. Por ello, los efectos sobre el desempleo y las tasas de pobreza fueron diferenciales en las tres macrorregiones del país, aquejando más intensamente a las entidades norteñas y centrales que a las sureñas, en las que en vez de acrecentarse el desempleo, se incrementaron las tasas de informalidad, paliando en alguna medida los efectos más nocivos de la crisis sobre la pobreza alimentaria. Como resultado, se registró una acusada convergencia de las tasas de pobreza alimentaria en las entidades federativas durante la etapa más severa de la recesión, sugiriendo un proceso regional de igualación por empobrecimiento, similar al encontrado por Cortés y Rubalcava (1991) a escala nacional en términos de la distribución del ingreso de los hogares.

En el primero de los seis apartados de este artículo se revisa la bibliografía disponible sobre la relación entre el ciclo económico, el mercado laboral y la evolución de la pobreza. El segundo contiene un breve examen del comportamiento de la economía mexicana durante las fases del ciclo registrado entre 2005 y 2012. En la tercera parte se describe la metodología para medir indirectamente las tasas trimestrales de pobreza alimentaria, tanto a escala nacional como de las entidades federativas del país, en tanto que en la cuarta se examina la trayectoria seguida por las tasas nacionales y estatales de pobreza alimentaria. El quinto apartado ofrece un análisis cuantitativo, mediante la estimación de varios modelos de regresión con un panel de datos, de la relación entre el mercado laboral y las tasas de pobreza en el contexto del ciclo económico, y el sexto constata sus tendencias convergentes entre las entidades federativas en la fase recesiva del ciclo. Finalmente, se ofrece una breve recapitulación de lo expuesto y se apuntan algunas implicaciones de política pública.

\section{ANTECEDENTES TEÓRICOS Y EMPÍRICOS}

Cuantificada de manera absoluta, la pobreza de los países tiende a disminuir en el largo plazo conforme aumenta el ingreso real per cápita, mientras que en el corto 
plazo varía con los movimientos de la actividad económica (Fields, 1980, p. 53). Es por ello que en la bibliografía contemporánea suele distinguirse la pobreza permanente, o de largo plazo, de la pobreza temporal, o de corto plazo. La movilidad de los hogares para entrar o salir de la pobreza permanente (también llamada estructural) está condicionada por el crecimiento de largo plazo de la economía; en contraste, en el corto y mediano plazos, sus posibilidades de movilidad están determinadas por las fases del ciclo económico, especialmente por las variaciones en la TDA (Blank y Blinder, 1985).

La economía más estudiada en este sentido es la de Estados Unidos. En ese país se ha detectado, de manera robusta, que la salida de episodios de pobreza por parte de los hogares no los conduce necesariamente a condiciones permanentes de no pobreza, sino que es usual su recurrencia en las siguientes fases depresivas del ciclo económico (Bane y Ellwood, 1986; Stevens, 1994). ${ }^{1}$ Tobin (1992), al analizar la evolución de la pobreza en la economía estadounidense, observó que su dramática disminución desde finales de la Segunda Guerra Mundial obedeció a la singular expansión del empleo registrada hasta principios de los setenta. Sin embargo, hizo notar que a partir de entonces el menor crecimiento del empleo y de los salarios reales ha provocado que la pobreza en EU se vincule más estrechamente a las recesiones cíclicas de su economía -cada vez más frecuentes a partir de los setenta-, las cuales se han acompañado de modificaciones muy poco favorables en la naturaleza de la política social. ${ }^{2}$

Algunos análisis indican que las expansiones económicas benefician en mayor grado a los hogares más pobres, por los efectos favorables que se derivan de la creación de empleo; sin embargo, otros muestran que en la economía estadounidense esa relación se ha atenuado desde finales de los ochenta, como consecuencia del estancamiento de la productividad y del aumento de la desigualdad salarial (Blank, Card, Levy y Meddof, 1993). Investigaciones paralelas en ese país precisan la naturaleza de este fenómeno. Por ejemplo, Hoynes (1999), mediante un análisis de regresión para el período 1975-1997, encuentra que las re-

\footnotetext{
${ }^{1}$ Stevens (1999) encuentra que en Eu la mitad de los hogares que salen de un episodio de pobreza regresarán de nuevo a esa condición en los siguientes cuatro o cinco años, una vez concluida la fase expansiva del ciclo económico.

${ }^{2}$ El nexo entre el ciclo económico y la pobreza se deriva de la vigencia de la ley de Okun, según la cual, los aumentos (o disminuciones) de la actividad económica se acompañan de disminuciones (o aumentos) de la TDA (Okun, 1962). Las desfavorables modificaciones de la política social mencionadas por Tobin sustituyeron las transferencias en dinero a los hogares en pobreza otorgadas en los sesenta por transferencias en cupones para adquirir alimentos y por el acceso a Medicaid, a partir de entonces.
} 
percusiones del ciclo sobre el mercado laboral y la pobreza son mayores en los hogares de trabajadores con bajos niveles educativos y escasa calificación, y conllevan notables sesgos de raza y sexo, que afectan más desfavorablemente a los negros y a las mujeres. Baulch y Hoddinott (2000) corroboran que la mayoría de los hogares entran y salen de la pobreza con los movimientos de la economía, y muestran además que las familias estadounidenses en pobreza transitoria afectadas por impactos temporales, enfrentan consecuencias muy importantes que determinan en buena medida la trayectoria de sus condiciones de pobreza en el largo plazo.

En los Estados Unidos, el fenómeno ha sido analizado también en términos regionales. Gundersen y Ziliak (2004), al examinar un panel de los 52 estados para el período 1981-2000, además de confirmar que una macroeconomía robusta reduce la tasa y la intensidad de la pobreza, encuentran que los efectos de las fases recesivas del ciclo son marcadamente diferentes entre hogares ubicados en distintas regiones y/o con diversas características demográficas. En una contribución más reciente, Hoynes, Page y Stevens (2006), con información anual de 1967 a 2003, analizan, también por medio de regresión con un panel de datos, las repercusiones del ciclo sobre la tasa de pobreza en los estados de ese país, y demuestran que las dos variables que se correlacionan más significativamente con aquélla en términos estadísticos son, con signo positivo, la TDA y, con signo negativo, la evolución de las remuneraciones laborales reales.

El análisis del fenómeno en las economías latinoamericanas es más escaso. De Janvry y Sadoulet (1999) examinan los efectos del crecimiento y la concentración de la distribución del ingreso en una muestra de países de la región, y además de confirmar que el primero abate y la segunda aumenta las tasas de pobreza, señalan la asimetría que se observa entre los episodios expansivos y contractivos del ingreso derivados de impactos externos, ya que un año de recesión en estos países anula las disminuciones de la pobreza acumuladas en dos o más años de crecimiento, sobre todo en el caso de la pobreza rural. En contraposición, los efectos provocados por la inflación y/o la devaluación tienen costos mucho mayores en términos de pobreza urbana. Mediante un análisis econométrico más complejo, Agénor (2004) corrobora los efectos asimétricos sobre los pobres de los impactos en la actividad económica generados durante el ciclo económico en Brasil.

Sin embargo, el caso mexicano ha sido poco profundizado en la bibliografía especializada. Al examinar con encuestas de hogares la evolución de largo plazo de la incidencia de la pobreza y mediante metodologías no siempre uniformes, 
las investigaciones consultadas reseñan de manera explícita las desfavorables secuelas de las recesiones más severas ocurridas en la segunda mitad del siglo pasado y la primera década del presente; ${ }^{3}$ empero, esas investigaciones no examinan cuantitativamente la relación entre las variaciones en la actividad económica y las tasas de pobreza.

Un poco más enfocado en el análisis cuantitativo de esa relación se encuentra Hernández Laos (2009), quien descompone los cambios de las tasas de pobreza entre 1992 y 2006, y cuantifica las perniciosas secuelas de la contracción de la economía en 1996, y de manera separada cuantifica los efectos (también desfavorables) del aumento de la desigualdad en la distribución del ingreso. En otra investigación, el mismo autor aplica el filtro Hodrick-Prescott a las tasas de pobreza para el período 1992-2006 y, descontando el efecto de la tendencia de mediano plazo, encuentra que las variaciones residuales se explican de manera estadísticamente significativa por el comportamiento de la tasa urbana de desempleo abierto, que sigue de cerca la trayectoria del ciclo económico de la economía mexicana (Hernández Laos, 2006, p. 22).

Quizás por la periodicidad de la Encuesta Nacional de Ingresos y Gastos de los Hogares (ENIGH), disponible cada dos años sólo desde 1992, es que hasta ahora no se ha analizado con mayor acuciosidad la relación entre el ciclo económico y la pobreza en México. Sin embargo, la disposición trimestral del ITLP, elaborado por el CONEVAL, a partir de 2005, ha permitido que recientemente fuera utilizado por Benítez (2012), con los ajustes que más adelante se discuten, para analizar a escala nacional y estatal este fenómeno, durante las fases del ciclo económico que antecedieron y siguieron a la crisis global de 2008-2009.

\section{EL CICLO ECONÓMICO EN LA PRIMERA DÉCADA DEL NUEVO SIGLO}

El crecimiento de la economía mexicana en los noventa no logró compensar la severidad del efecto Tequila de 1995-1996 y el registrado en la primera década del nuevo siglo tampoco fue particularmente dinámico, a consecuencia del des-

\footnotetext{
${ }^{3}$ Sobre los efectos de la crisis de 1982-1983, véase Hernández Laos (1992, pp. 197-122); sobre los de la recesión de 1995-1996, Cortés et al. (2003, pp. 18-19), Székely (2003, p. 47), Hernández Laos (2003, pp. 109-111) y Hernández Laos y Velázquez (2003, pp. 75-101); y sobre los de la crisis más reciente de 2008-2009, Aparicio (2009, pp. 24-25) y Cortés (2010, pp. 77-86); las dos últimas investigaciones basan su análisis en estimaciones de pobreza por ingreso del CONEVAL, a partir de la metodología originalmente propuesta por el Comité Técnico para la Medición de la Pobreza (2002).
} 
favorable contexto económico internacional caracterizado por la recesión estadounidense de 2001-2002 y, años más tarde, por la gran recesión de 2008-2009, cuyos efectos resentimos hasta ahora. ${ }^{4}$

Iniciada en diciembre de 2007, la recesión en EU concluyó técnicamente en junio de 2009; sin embargo, hasta ahora la recuperación de su economía ha tenido escasos efectos sobre la disminución de la tasa oficial de pobreza, que aumentó de $12.5 \%$ en 2007 a 15\% en 2011, conforme crecía el desempleo abierto de largo plazo en ese país (Seefeldt et al., 2012, p. 5), confirmando así la relación entre el ciclo económico y la pobreza.

En contraste, en los países en desarrollo y emergentes, los efectos recesivos fueron poco significativos y/o inexistentes, sea porque en la década anterior desarrollaron un creciente comercio bilateral y/o porque tuvieron una menor exposición a los riesgos de la ingeniería financiera instrumentada en EU durante los años expansivos que precedieron a la crisis. De hecho, en varias de estas economías el desempleo no sólo no aumentó, sino se redujo y, de acuerdo con las estimaciones del Banco Mundial, la incidencia de la pobreza disminuyó en la etapa más severa de la crisis. ${ }^{5}$

Ése fue el caso también en la mayor parte de los países latinoamericanos, que disminuyeron sus tasas de pobreza -no sólo mediante los austeros criterios del Banco Mundial (2007-2010), sino también con metodologías más apropiadas a sus niveles de vida, como las elaboradas por el Centro de Estudios Distributivos, Laborales y Sociales (CEDLAs) de la Universidad Nacional de La Plata (2003-2009) - tanto en la región en su conjunto, como en la mayoría de los

\footnotetext{
${ }^{4}$ Como se sabe, la laxitud en el otorgamiento de crédito, la introducción de nuevos y no probados instrumentos financieros, la creciente desregulación bancaria y crediticia y, especialmente, el florecimiento de la ideología neoliberal en la economía estadounidense provocaron el auge generalizado de los mercados bursátiles e inmobiliarios desde el inicio de los noventa. Al estallar en EU la burbuja especulativa en 2007-2008, resultó inevitable el colapso de los precios de las viviendas, el masivo incumplimiento en el pago de hipotecas y las cuantiosas pérdidas producidas por el derrumbe de las cotizaciones bursátiles, todo lo cual provocó severas restricciones crediticias y aumentos en las tasas de interés exigidas por un sistema financiero en bancarrota, incapaz de cubrir los crecientes riesgos derivados de la crisis (Stiglitz, 2010, pp. 1-26; Ekes, 2011, pp. 191-219). El colapso de la economía estadounidense se propagó rápidamente, afectando los mercados financieros, bursátiles e inmobiliarios de la mayor parte de los países desarrollados, arrastrándolos a una profunda recesión que acrecentó el desempleo a escala mundial (OIT, 2011, pp. 12-16).

${ }^{5}$ Como en los casos de China, Filipinas, India, Indonesia, Malasia, Rumania, Turquía y Sudáfrica, considerando ingresos menores a USD 1.25 y USD 2 diarios por persona, valuados a la paridad del poder adquisitivo (PPP) (Banco Mundial, World Development Indicators, base de datos en $\mathrm{CD}$ ).
} 
países del área para los que se cuenta con información en el año previo al estallido de la crisis (2007) y en el último año disponible (2009 y/o 2010). ${ }^{6}$

Empero, eso no fue lo que sucedió con la economía mexicana, que se vio severamente afectada por la crisis estadounidense ya desde el primer trimestre de 2008. La gravedad de las repercusiones obedeció a tres factores principales: la disminución de las remesas del exterior, la reducción de los flujos de capital foráneo y, especialmente, la contracción y el dislocamiento de nuestras exportaciones (Griffith-Jones y Ocampo, 2009, pp. 2-4). ${ }^{7}$ La severidad de la recesión no tardó en dejarse sentir sobre la economía real de nuestro país, la cual venía desacelerando su crecimiento desde 2006, situación que se acentuó en 2007, con el inicio de la contracción en algunos sectores desde principios de 2008, y se prolongó hasta el cuarto trimestre de 2009 (gráfica 1). Con fines analíticos, en el cuadro 1 se traza una calendarización aproximada de las tres fases del ciclo de la economía mexicana en la segunda parte de la década pasada y los primeros dos años de la presente, tomando como indicador el crecimiento anual del producto interno bruto (PIB), total y sectorial, en términos reales. ${ }^{8}$

En forma esquemática, podemos identificar una fase de expansión moderada, que cubre desde el primer trimestre de 2006 hasta el cuarto trimestre de

\footnotetext{
${ }^{6}$ Las estimaciones del CEDLAS toman como línea de pobreza cuatro dólares por día, ajustados por la PPP, sobre bases uniformes en los países de la región. A partir de ello, se estima que entre 2003 y 2009 la tasa de pobreza en el conjunto de la región se redujo en $11.9 \%$, lo que en términos absolutos significó una disminución de 49.2 millones de personas en condición de pobreza en países como Brasil, Chile, Colombia, Ecuador, Perú, Argentina, República Dominicana y Uruguay (CEDLAS-UNLP y Banco Mundial, 2011).

${ }^{7}$ Las remesas procedentes de EU, muy significativas en términos de recursos externos, se contrajeron en cerca de $20 \%$ entre 2007 y 2009 , y para 2012 no habían recuperado el nivel que tenían antes de la crisis (Fundación BBva Bancomer, 2013). En 2009, la inversión extranjera directa (IED) se había reducido a la mitad en relación a la registrada en 2007, y para 2011 mostraba todavía niveles $35 \%$ menores que los alcanzados cuatro años antes, a pesar de la impetuosa entrada de capital en cartera, que para esas mismas fechas había triplicado el nivel previo a la crisis (Presidencia de la República, 2012). Con todo, el más notable abatimiento de los ingresos foráneos se dio, sin duda, en el comercio exterior, dada la vinculación de nuestras exportaciones de crudo y de productos industriales intermedios con la economía estadounidense, cuya demanda se redujo de manera notable desde mediados de 2008 (Levchenko, Lewis y Tesar, 2010, p. 30).

${ }^{8}$ Aunque en un sentido técnico la recesión en México se inició en el cuarto trimestre de 2008 y se manifestó especialmente severa en los siguientes tres trimestres de 2009, los efectos contractivos no fueron homogéneos en términos sectoriales, ya que desde principios de 2008 se hicieron notar en las actividades terciarias, en tanto que en el sector secundario los efectos recesivos no comenzaron a manifestarse hasta el tercer trimestre de 2008, y en el sector primario la contracción se presentó entre el primer trimestre de 2009 y el primero de 2010 (INEGI, 2012a).
} 
Gráfica 1. México. Tasa de crecimiento medio anual del PIB real por trimestres, $2005_{1}$ a $2012_{4}$

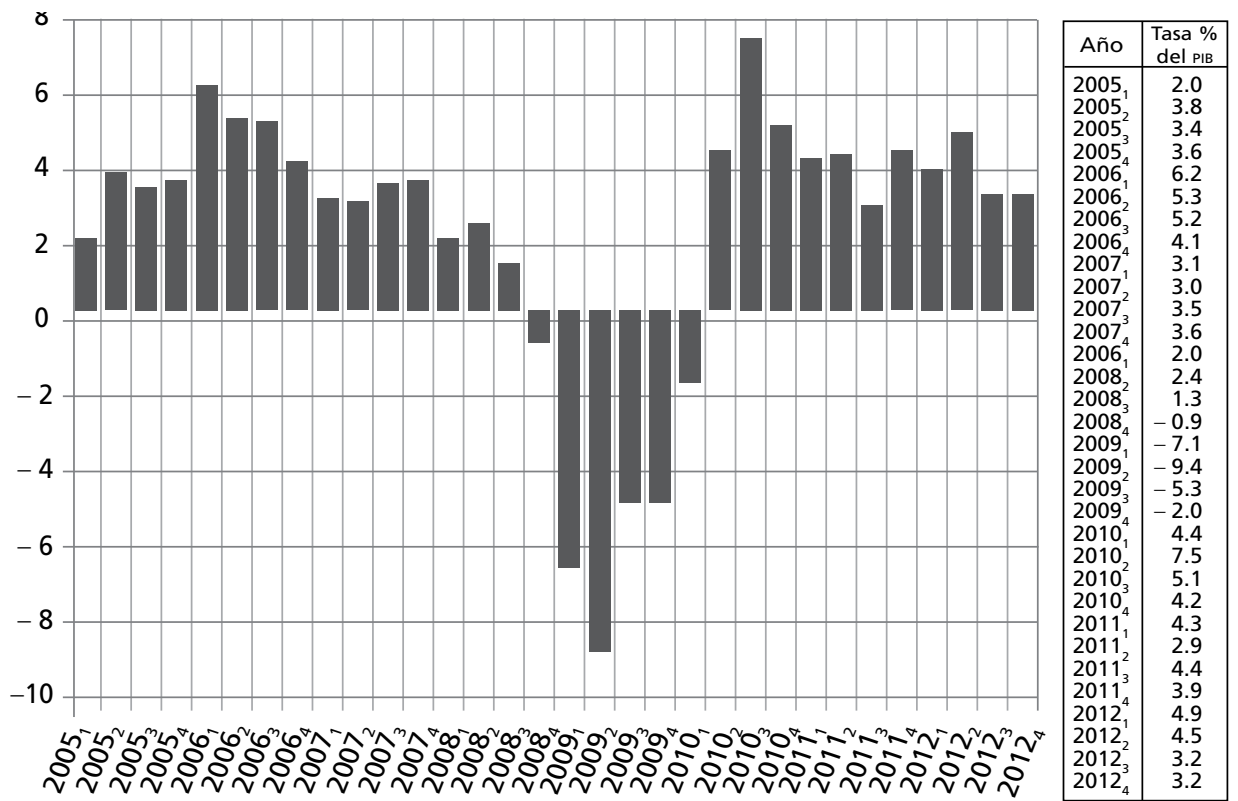

Tasa porcentual del PIB

Nota: Los subíndices indican trimestres del año respectivo.

Fuente: Cálculos propios con información del Sistema de Cuentas Nacionales de México, INEGI (varios años).

2007, con tasas de crecimiento positivas aunque decrecientes para un promedio de $4.2 \%$ anual. Una segunda fase, de naturaleza contractiva, empezó en el primer trimestre de 2008, al interrumpirse el crecimiento del sector terciario, y se prolongó hasta el cuarto trimestre de 2009, con el descenso de la producción de los sectores primario y secundario.

En los ocho trimestres que duró la fase recesiva, la economía mexicana decreció a un promedio anual de $2.4 \%$, resultando el sector más afectado, sin duda, el secundario, cuya contracción fue cercana a $4 \%$ anual. Por último, a partir del primer trimestre de 2010 se inició una etapa de recuperación paulatina que se prolongó hasta el cuarto trimestre de 2012, registrándose un crecimiento anual promedio de $4.6 \%$ (cuadro 1 ).

El colapso de la producción en la fase contractiva del ciclo económico afectó severamente el desempeño del mercado laboral mexicano. En efecto, en el contexto del enérgico crecimiento de la oferta laboral, producto de la profundiza- 


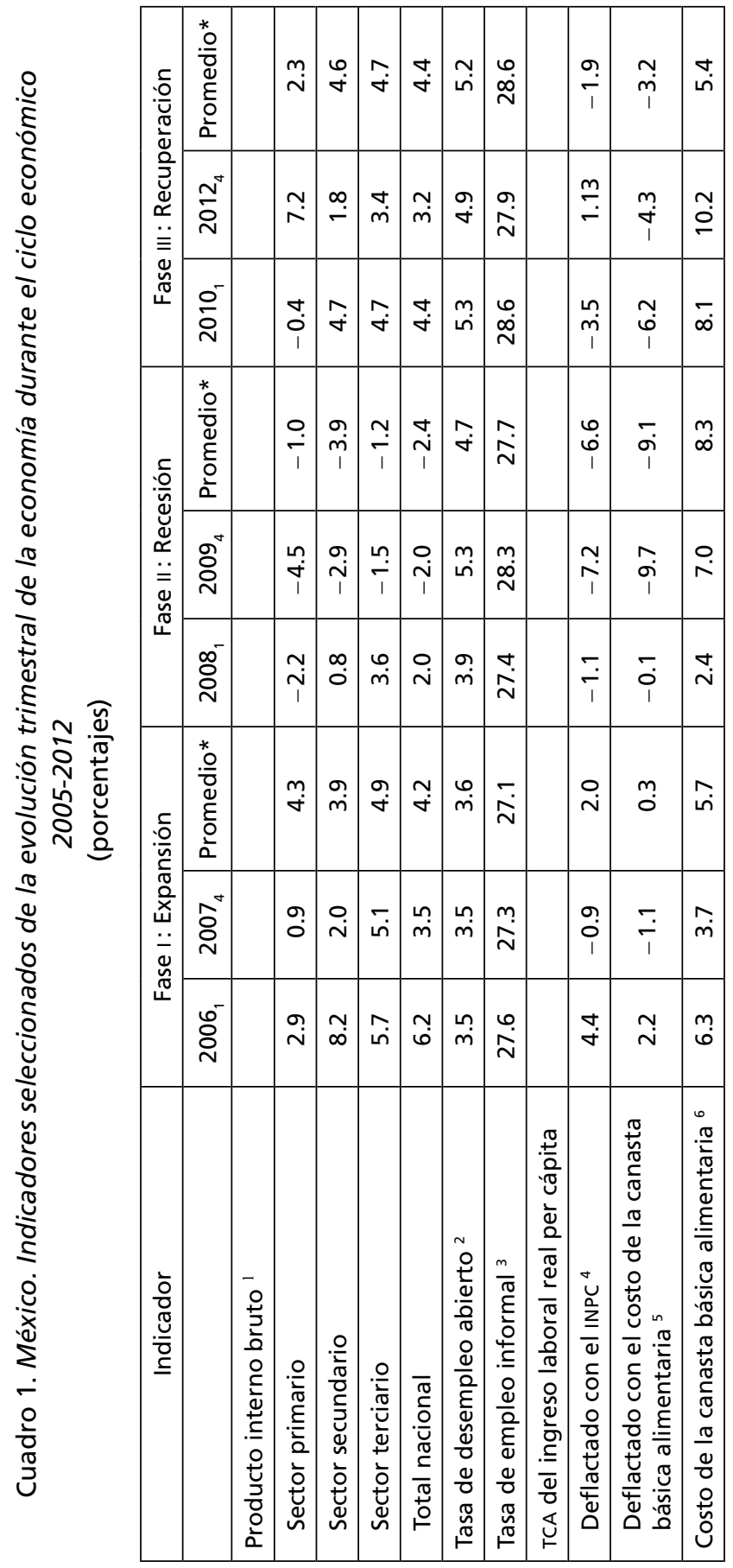

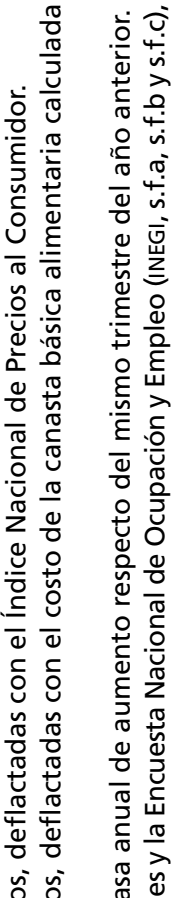

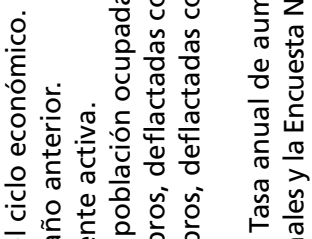

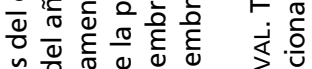

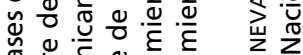

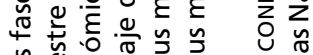

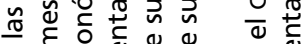

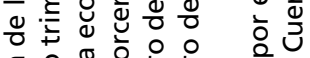

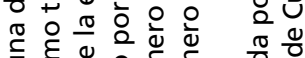

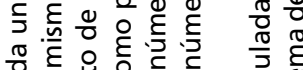

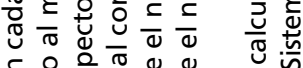

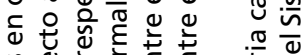
นิ

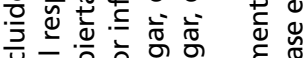

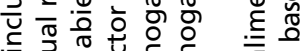

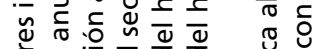

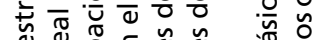

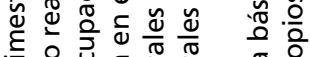

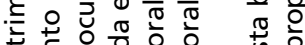

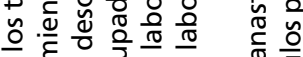

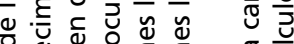

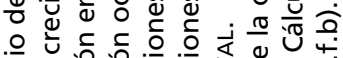
원 은 은 는 ह

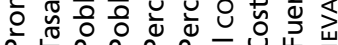

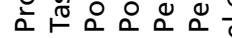


ción del cambio demográfico, el mercado de trabajo redujo la demanda de mano de obra en la etapa recesiva y la estancó en los sectores primario y terciario durante la recuperación (Hernández Laos, Solís y Stefanovich, 2013, cap. 2). ${ }^{9}$ En consecuencia, desde 2008 se intensificaron los desequilibrios del mercado laboral, acrecentándose la TDA y la tasa de ocupación informal y, aunque se estancaron los salarios diarios reales del sector formal, el mayor subempleo acentuó el deterioro de los ingresos reales per cápita, que fue particularmente severo al deflactarse los ingresos nominales por el costo de la canasta básica alimentaria estimada por el CONEvAL (cuadro 1). ${ }^{10}$

Los efectos de la crisis sobre la actividad productiva y el mercado laboral fueron desiguales en las tres macrorregiones en que suele dividirse el país (Bassols, 1971): fueron mayores en las entidades norteñas y centrales que en las ubicadas en el sur-sureste del país, dadas las diferentes estructuras productivas que las caracterizan. ${ }^{11}$ Durante la etapa expansiva del ciclo, las entidades del

${ }^{9}$ Entre 2007 y 2012, los nuevos entrantes a la fuerza de trabajo sumaron 6.5 millones de personas, de las cuales sólo 5.5 millones obtuvieron un puesto productivo, quedando en el desempleo abierto más de un millón de mexicanos económicamente activos. Destaca la precariedad del empleo generado si se considera que poco más de dos terceras partes de estos entrantes se ocuparon en microestablecimientos y/o en condiciones de autoempleo, parte importante de ellos sin remuneración económica alguna (Presidencia de la República, 2012).

${ }^{10}$ En la fase de expansión, la TDA se mantuvo en alrededor de $3.6 \%$ de la población económicamente activa (PEA); sin embargo, pasó de 3.9\% al inicio de la fase recesiva a 5.3\% al concluirse, y se mantuvo elevada y ligeramente creciente a lo largo de la recuperación, del primer trimestre de 2010 hasta el cuarto de 2012. Una dinámica similar observó la TOSI, que se acrecentó a lo largo de las tres fases del ciclo, hasta alcanzar 28.2\% de la ocupación no agropecuaria a finales de 2012 (cuadro 1). El deterioro de las remuneraciones laborales reales fue particularmente severo: de $-7.2 \%$ promedio anual durante los ocho trimestres de la etapa recesiva, una vez deflactadas las remuneraciones nominales con el Índice Nacional de Precios al Consumidor (INPC), y de $-9.7 \%$, con el costo de la canasta básica alimentaria del CONEval. La pérdida del poder adquisitivo continuó durante toda la etapa de recuperación hasta finales de 2012 (cuadro 1). En una investigación reciente (Campos-Vázquez, 2013) se sostiene que esas cuantificaciones podrían ser no muy precisas, si se tiene en cuenta el aumento en los últimos años reportado por la Encuesta Nacional de Ocupación y Empleo (ENOE) del número de trabajadores remunerados que no declara ingresos, lo que sesga hacia la baja el promedio per cápita de los hogares en los últimos trimestres; si se incluyen los valores omitidos, el "verdadero" (sic) ingreso familiar per cápita no mostraría cambios sustanciales en el período y reduciría las estimaciones de la incidencia de la pobreza laboral del CONEVAL, que son las que utilizamos más adelante en nuestra investigación.

${ }^{11}$ Con base en el comportamiento trimestral del Indicador Global de la Actividad Económica (IGAE) de las 32 entidades federativas del país (INEGI, s.f.c). La agregación de las tres macrorregiones consiste en promedios simples de las entidades que las componen, al igual que la agregación de la TDA y la TOSI. 
norte $^{12}$ y el centro ${ }^{13}$ registraron un crecimiento relativamente dinámico (mayor que $4.5 \%$ anual), mientras las ubicadas en el sur-sureste ${ }^{14}$ se desenvolvieron con menor celeridad (1.4\% anual) a consecuencia del escaso crecimiento de sus sectores primario y secundario (cuadro 2).

Durante la etapa recesiva, por el contrario, fueron las macrorregiones norte y centro las más severamente afectadas por la contracción económica ( $-2.4 \%$ y $-1.1 \%$ promedio anual), sobre todo en sus actividades secundarias (mineras y manufactureras), en tanto que la sur-sureste registró un crecimiento positivo (1.1\% promedio anual), a pesar de la severa contracción de sus actividades secundarias (-5.4\% promedio anual) (cuadro 2$)$.

Por último, en los doce trimestres de la recuperación, la expansión económica resultó más dinámica en el centro (5\% promedio anual) y el norte $(4.3 \%$ promedio anual), que en el sur-sureste (3.5\% promedio anual). La expansión de las primeras dos macrorregiones obedeció al pujante crecimiento del sector secundario, especialmente en el centro, en tanto que en el sur-sureste el crecimiento de las actividades primarias y las terciarias no logró compensar la escasa recuperación de las secundarias (cuadro 2).

En su conjunto, a lo largo de los 32 trimestres comprendidos en las tres fases del ciclo, el centro del país resultó ser el menos afectado por la severidad de la recesión, dado que su crecimiento medio anual (3.1\%) fue mayor que el alcanzado por el norte $(2.7 \%)$, en tanto que el sur-sureste prolongó en el mismo lapso su tradicional rezago en materia de crecimiento económico $(2.2 \%)$.

\section{EL DESEMPEÑo DEL MERCADO LABORAL EN LAS FASES DEL CICLO}

En contraste, las repercusiones regionales sobre el mercado laboral fueron menos heterogéneas. Por ejemplo, en el sur-sureste, la TDA, aunque ligeramente creciente, se mantuvo baja durante las tres fases del ciclo (entre $2 \%$ y $3 \%$ ), mientras que en el norte y el centro el desempleo aumentó entre uno y dos puntos porcentuales durante la recesión, y continuó incrementándose ya en la recuperación, aunque menos dinámicamente, para alcanzar en ambas macrorregiones niveles

\footnotetext{
${ }^{12}$ Baja California, Baja California Sur, Chihuahua, Coahuila, Nayarit, Nuevo León, Sinaloa, Sonora y Tamaulipas.

${ }^{13}$ Aguascalientes, Zacatecas, Veracruz, Tlaxcala, Tabasco, San Luis Potosí, Querétaro, Puebla, Morelos, Michoacán, Estado de México, Jalisco, Hidalgo, Guanajuato, Durango, Distrito Federal y Colima.

${ }^{14}$ Campeche, Chiapas, Guerrero, Oaxaca, Quintana Roo y Yucatán.
} 


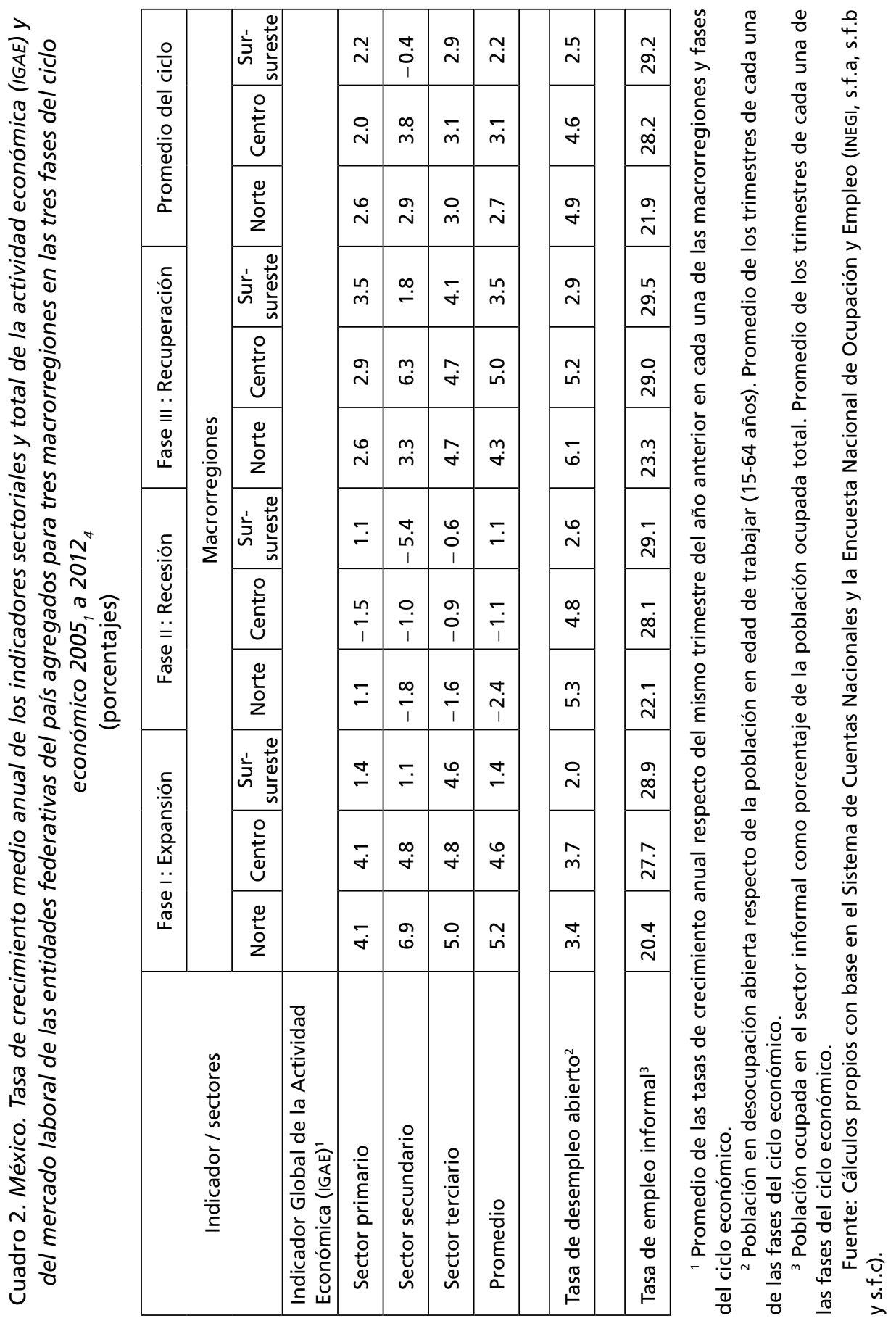


elevados para los estándares de nuestro mercado laboral, de entre $5 \%$ y $6 \%$ en promedio (cuadro 2).

Además, y dada la ausencia de un seguro de desempleo universal en México, el mercado laboral registró, de acuerdo con las cuantificaciones del Instituto Nacional de Estadística y Geografía (INEGI), ${ }^{15}$ aumentos de la ocupación informal no sólo durante la fase depresiva del ciclo, sino también en los trimestres de la recuperación. Así, y aunque la Tosi creció en toda la geografía nacional, los incrementos fueron mayores en el centro y el sur-sureste, que hacia finales del ciclo alcanzaron niveles cercanos a $30 \%$, en tanto que el norte no alcanzó cotas tan elevadas, toda vez que en esa macrorregión el aumento se registró más bien en la tasa de desempleo abierto (cuadro 2).

En resumen, y al contrario de lo acontecido en la mayor parte de los países latinoamericanos, en México los efectos de la recesión económica inter-

\section{Cuadro 3. México. Tasa de pobreza y prueba de hipótesis sobre la significación estadística del cambio en la incidencia de la pobreza, 2006 - $2012^{1}$}

\begin{tabular}{|c|c|c|c|c|c|c|c|c|c|}
\hline \multirow{2}{*}{$\begin{array}{c}\text { Línea de } \\
\text { pobreza }\end{array}$} & \multicolumn{2}{|c|}{$\begin{array}{c}\text { Tasa de } \\
\text { pobreza } \\
(\%)\end{array}$} & \multicolumn{2}{|c|}{$\begin{array}{c}\text { Error } \\
\text { estándar } \\
(\%)\end{array}$} & \multirow[t]{2}{*}{$\begin{array}{c}\text { Diferencia } \\
\text { tasa de } \\
\text { pobreza }\end{array}$} & \multirow[t]{2}{*}{$\begin{array}{c}\text { Error } \\
\text { estándar } \\
\text { de la } \\
\text { diferencia }\end{array}$} & \multirow[t]{2}{*}{$\begin{array}{c}\text { Estadístico } \\
Z\end{array}$} & \multirow[t]{2}{*}{$\begin{array}{c}\text { Nivel de } \\
\text { significación }\end{array}$} & \multirow[t]{2}{*}{$\begin{array}{l}\text { Significación } \\
\text { estadística }\end{array}$} \\
\hline & 2006 & 2012 & 2006 & 2012 & & & & & \\
\hline $\begin{array}{l}\text { Pobreza } \\
\text { alimentaria }\end{array}$ & 13.8 & 20.0 & 0.7306 & 0.4360 & 6.18 & 1.13 & 4.43 & 0.0000 & Significativa \\
\hline $\begin{array}{l}\text { Pobreza de } \\
\text { patrimonio }\end{array}$ & 42.7 & 51.6 & 0.8311 & 0.3600 & 8.94 & 0.83 & 10.34 & 0.0000 & Significativa \\
\hline
\end{tabular}

${ }^{1}$ Las estimaciones de 2006 utilizan factores de expansión ajustados a los resultados definitivos del Censo de Población y Vivienda de 2010 y las de 2012 a los de la ENIGH-2012.

${ }^{2}$ Las pruebas de hipótesis son de dos colas, con un nivel de significación de 0.05 .

Fuente: Estimaciones del CONEVAL (s.f.b) con base en la ENIGH 2006 y 2012.

${ }^{15}$ En su Sistema de Cuentas Nacionales por Sectores Institucionales, el INEGI presenta estimaciones de la ocupación en el sector informal, cuantificada desde la óptica productiva, esto es, ocupados en micronegocios y en unidades no registradas. Recientemente esa institución, siguiendo las recomendaciones de la Organización Internacional del Trabajo (OIT), amplió su definición de informalidad, y actualmente también incluye los trabajadores asalariados a los que no se les reconoce la totalidad de sus derechos laborales, es decir, los no registrados en las instituciones de seguridad social del país. En los análisis aquí presentados se recurre a la Tosi, la cual utiliza el concepto de ocupación informal previo a la recomendación de la oIT, por lo cual no incluye a los asalariados sin seguridad social. El indicador del INEGI que sí los incorpora se denomina tasa de informalidad laboral (TIL) (INEGI, Boletín de prensa Núm. 449/12; 11 de diciembre de 2012). Para una discusión in extenso de la metodología aplicada en las nuevas mediciones, véase: INEGI (2012b) e ILO (2012). 
nacional de 2008-2009 fueron más severos y provocaron desajustes de consideración en el mercado laboral, acrecentando el desempleo abierto y la ocupación informal. Tales desajustes redujeron las remuneraciones reales de los hogares y, al deteriorar su poder adquisitivo en términos de la canasta básica alimentaria del CONEVAL, acrecentaron de manera estadísticamente significativa las tasas de pobreza alimentaria que, con un criterio de continuidad y comparación con años anteriores, había calculado el CONEVAL para el periodo comprendido entre 2006 y 2012 (cuadro 3 ).

\section{ESTIMACIONES TRIMESTRALES DE LAS TASAS DE POBREZA ALIMENTARIA}

Además de las estimaciones oficiales de pobreza calculadas por el CONEVAL con información de la ENIGH, esa institución calcula trimestralmente desde 2005 el ITLP, con información sobre ingresos laborales de la ENOE. La nota metodológica que sustenta esas estimaciones explica que, dada la correlación inversa entre los cambios en la masa salarial y los cambios en la pobreza por ingresos observada entre 1992 y 2008 (Hernández y Székely, 2009), puede suponerse que se mantiene el comportamiento de espejo entre la masa salarial (salarios por empleo) y la pobreza laboral, siempre y cuando ambas variables se midan en términos estandarizados (CONEVAL, 2010, pp. 17-18). Extendiendo el razonamiento, y dado que los ingresos laborales representan alrededor de dos terceras partes del ingreso corriente total y cuatro quintas partes del ingreso corriente monetario de los hogares (Benítez, 2012, p. 21), podemos asumir que se mantiene en el tiempo la correlación entre las tasas oficiales de pobreza alimentaria y las mediciones realizadas a partir del ingreso laboral de los hogares. ${ }^{16}$

Con esa lógica, en lo que sigue utilizamos los informes del ITLP procesados por el CONEVAL tanto a escala nacional como de las 32 entidades federativas del país, para medir indirectamente la evolución trimestral de las tasas nacionales y estatales de pobreza alimentaria. En la medida en que el ITLP identifica la evolución del porcentaje de la población que no puede adquirir la canasta alimentaria con el ingreso de su trabajo, esa proporción queda establecida por el desempeño del mercado laboral y por los aumentos en la línea de pobreza determinada por el

${ }^{16}$ El Coneval (2012, p. 20) encuentra un coeficiente de correlación positivo y altamente significativo $(r=+0.98 ; p=0.01)$ entre las tasas de pobreza alimentaria calculadas con el ingreso laboral de los hogares y las estimadas con base en el ingreso corriente total de los hogares. 
costo de la canasta alimentaria. El coneval conforma índices que toman como base el segundo trimestre de 2005, período inicial de la serie, y valores crecientes (o decrecientes) en los trimestres siguientes, mediciones que se prolongan hasta el cuarto trimestre de 2012.

Por construcción, sabemos que la evolución del ITLP no necesariamente debe coincidir con la de las tasas oficiales de pobreza alimentaria, en la medida en que aquél no toma en cuenta los ingresos no laborales, cuya cuantía puede ser relevante para la medición de la pobreza alimentaria, ni las transferencias recibidas por los hogares en forma de remesas del exterior (Esquivel y Huerta-Pineda, 2007) ni las originadas por los programas vigentes de combate a la pobreza (Cortés, Banegas y Solís, 2007). ${ }^{17}$ Sin embargo, dado que la fracción más relevante de los ingresos de los hogares la constituyen los ingresos laborales y con el propósito de simular la evolución trimestral de la pobreza alimentaria entre 2005 y 2012, supondremos que la variación trimestral de la TPA a nivel nacional y de las 32 entidades federativas puede medirse indirectamente de manera satisfactoria si las tasas oficiales de inicio del periodo (segundo trimestre de 2005$)^{18}$ se extrapolan para los siguientes trimestres, aplicando la evolución trimestral del ITPL nacional y de cada entidad, con el objeto de analizar su relación con los indicadores del mercado laboral (TDA y TOSI) en las diversas fases del ciclo económico.

Vale hacer hincapié, sin embargo, en que estas extrapolaciones no necesariamente coinciden con las estimaciones oficiales del CONEVAL, especialmente en 2006, cuando la tasa oficial de pobreza alimentaria fue de sólo $13.8 \%$, en tanto que la proyectada con esta metodología alcanzó $17.9 \%$, es decir, 4.1 puntos porcentuales mayor, a pesar de que en los siguientes años para los que se cuenta con información oficial la diferencia es menor ( 0.5 puntos porcentuales más en 2008 y 2.8 puntos porcentuales en 2012), en los que si bien no se elimina la desviación, sí se reduce de manera apreciable. ${ }^{19}$ Los sesgos descritos se reflejan en

${ }^{17}$ Para dimensionar lo anterior, considérese que las transferencias recibidas por los hogares aumentaron casi tres puntos porcentuales como proporción del ingreso corriente total de los hogares, al pasar de $8.6 \%$ en 2005 a 11.4\% en 2010 (Benítez, 2012, p. 21). Dado que una parte de las transferencias son recibidas por los hogares con menores ingresos, su efecto sobre la medición de las tasas de pobreza alimentaria puede ser importante en términos estadísticos, aunque con posibles diferencias entre las áreas rurales y las urbanas.

${ }^{18}$ Calculadas por el coneval (2010) con información del Conteo de Población y Vivienda de ese año realizado por el INEGI.

${ }^{19}$ Como se mencionó, el sesgo obedece, en parte, a que el ITPL no incluye las transferencias recibidas por los hogares (remesas y programas para el combate a la pobreza), como lo corrobora con el signo y significación estadística del coeficiente de correlación de Spearman entre las tasas trimestrales de pobreza alimentaria así estimadas, y la evolución trimestral de las remesas recibi- 
Cuadro 4. Ecuaciones de regresión entre las tasas oficiales de pobreza alimentaria del CONEVAL por entidad federativa con base en la ENIGH y las estimaciones propias a partir de los índices de pobreza laboral del CONEVAL

\begin{tabular}{|c|c|c|c|}
\hline Variable & ENIGH 2005 & ENIGH 2008 & ENIGH 2012 \\
\hline$\beta_{1}$ & 0.259 & & \\
\hline$\beta_{2}$ (PL05) & $0.983^{1}$ & & \\
\hline$\beta_{1}$ & & $1.75^{1}$ & \\
\hline$\beta_{2}$ (PL08) & & $0.846^{1}$ & \\
\hline$\beta_{1}$ & & & $4.229^{1}$ \\
\hline$\beta_{2}(\mathrm{PL} 12)$ & & & $0.764^{1}$ \\
\hline \multicolumn{4}{|c|}{ Estadísticos } \\
\hline$R^{2}$ & 0.996 & 0.883 & 0.823 \\
\hline SER & 0.677 & 3.411 & 4.099 \\
\hline Función de verosimilitud & -31.909 & -83.629 & -89.517 \\
\hline DW & 2.097 & 1.827 & 1.718 \\
\hline $\begin{array}{l}\text { Criterio de información } \\
\text { de Akaike }\end{array}$ & 2.119 & 5.352 & 5.719 \\
\hline Criterio de Schwart & 2.211 & 5.443 & 5.812 \\
\hline Hannan-Quin & 2.149 & 5.3822 & 5.75 \\
\hline$F$ (prob.) & $8126(0.0000)$ & $226.8(0.0000)$ & $139.6(0000)$ \\
\hline Muestra ${ }^{2}$ & 32 & 32 & 32 \\
\hline \multicolumn{4}{|c|}{ Prueba de Wald $\left(\beta_{1}=0 ; \beta_{2}=1\right)$} \\
\hline$F(2,30)$ & 1.250 & 5.191 & 7.072 \\
\hline$\chi^{2}(2)$ & 2.500 & 10.381 & 14.143 \\
\hline Hipótesis nula ${ }^{3}$ & Aceptada & Rechazada & Rechazada \\
\hline
\end{tabular}

${ }^{1}$ Diferente de 0 a 0.01 de probabilidad.

${ }^{2}$ Entidades federativas.

${ }^{3}$ Hipótesis nula: $\beta_{1}=0 ; \beta_{2}=1$.

Nota: Variable dependiente: tasa oficial de pobreza (CONEVAL con base en datos de la ENIGH para 2005, 2008 y 2012). Variable independiente: promedio anual de estimaciones trimestrales aplicando los índices de pobreza laboral del CONEVAL con la metodología explicada en el texto para los mismos años.

das por los hogares nacionales reportadas por Banco de México $(r=-0.7288 ; p=0.01)$, lo que podría explicar que en 2006 las remesas fueron cuantiosas. Como también se hizo notar más arriba, otra fuente de sesgo podría derivar de la omisión de ingresos de los hogares de la ENOE analizada por Campos-Vázquez (2012), quien muestra que al incluir los ingresos omitidos, se tendrían menores estimaciones de la pobreza laboral que las del coneval en los últimos trimestres de la serie. 
las estimaciones de la pobreza alimentaria de las entidades federativas, las cuales muestran desviaciones respecto de los cálculos oficiales de la pobreza alimentaria que se amplían marginalmente conforme se alejan del periodo base de estimación (cuadro 4). ${ }^{20}$ En resumen, las mediciones indirectas trimestrales, aunque no necesariamente invalidan las tendencias seguidas por la pobreza alimentaria a lo largo del ciclo económico, presentan sesgos que deberán tenerse presentes en los análisis que se ofrecen a continuación.

\section{El CICLO ECONÓMICO Y LOS CAMBIOS TRIMESTRALES DE LA POBREZA ALIMENTARIA}

La gráfica 2 muestra la evolución trimestral de la TPA estimada a nivel nacional. Se observa que después de la variabilidad registrada entre 2005 y 2007, fue a principios de 2008, al resentirse las primeras manifestaciones de la fase recesiva en los sectores primario y terciario, que la TPA aumentó de manera abrupta y sostenida hasta el último trimestre de 2009 , incrementándose en casi tres puntos porcentuales, al pasar de $18.4 \%$ a $21.3 \%$ de la población nacional. Superada dicha fase a principios de 2010, la pobreza alimentaria continuó aumentando, aunque menos aceleradamente, hasta el tercer trimestre de 2012, a pesar de que la economía creció $4.6 \%$ anual en promedio durante los doce trimestres que se prolongó la fase de recuperación (gráfica 2 y cuadro 5).

En las entidades federativas, la TPA siguió tendencias muy similares a la nacional. Con fines descriptivos, calculamos promedios simples de las tasas trimestrales en las entidades de las tres macrorregiones que venimos analizando (gráfica 3). Destacan con claridad los notables contrastes en su nivel, determinados por factores económicos y sociales estructurales de larga data, que provocan índices de pobreza alimentaria más elevados en las entidades del sur-sureste que en las centrales y las norteñas. Sin embargo, pese a los contrastes absolutos en su

\footnotetext{
${ }^{20}$ La cuantía del sesgo en las entidades se advierte mediante la estimación de ecuaciones de regresión de sección cruzada entre las tasas oficiales de pobreza alimentaria (ENIGH) y nuestras estimaciones de pobreza ( $P L)$ : $E N I G H X X_{j}=\beta_{1}+\beta_{2} * P L X X_{j}$ en cada uno de los años $(2005,2008$ y 2012). El cuadro 4 muestra que las tres regresiones son estadísticamente significativas $(p=0.05)$, y aunque los parámetros tienen el signo esperado, no cumplen con los valores requeridos para garantizar la igualdad de las tasas de pobreza alimentaria estimadas con ambas metodologías, como se aprecia mediante la prueba de Wald, cuya hipótesis nula: $\beta_{1}=0$ y $\beta_{2}=1$ se acepta sólo en 2005 y se desecha en 2008 y $2012(p=0.05)$. En conjunto, nuestras estimaciones subestiman marginalmente las tasas de pobreza alimentaria de los estados más desarrollados y sobreestiman, también marginalmente, las de las entidades con mayor rezago económico.
} 
Gráfica 2. México. Evolución trimestral de la TPA nacional estimada a partir de índices de pobreza laboral, 2005, a 2012 4

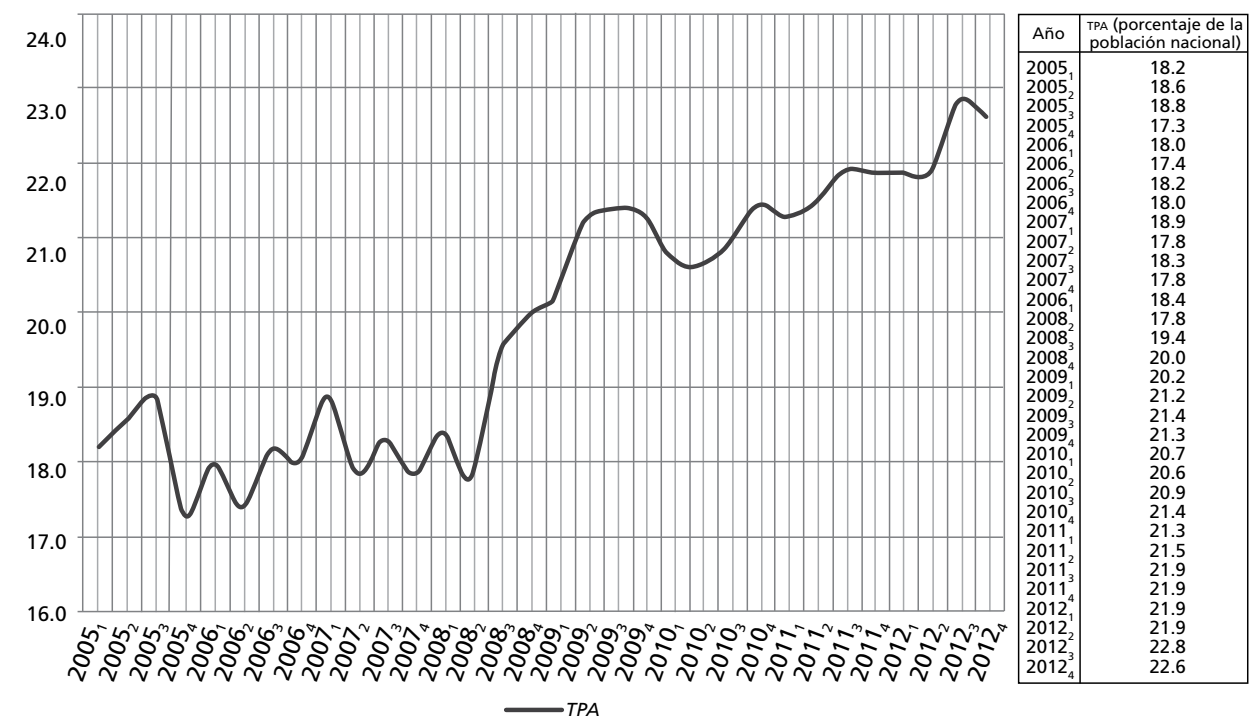

Fuente: Cálculos propios con la metodología explicada en el texto.

evolución, lo que interesa examinar es que adoptó perfiles muy similares en las tres macrorregiones, no sólo en términos absolutos de cada trimestre, sino también en términos incrementales entre trimestres, a lo largo de todo el ciclo económico. ${ }^{21}$

A pesar de la similitud de la evolución trimestral de la tasa de pobreza en las tres macrorregiones, el aumento registrado durante la fase recesiva fue mayor en las entidades centrales (3.2\%) que en las norteñas (2.4\%) y que en los estados más pobres, es decir, en los del sur-sureste de México (2.3\%). Como ya se mencionó, el incremento de la pobreza se prolongó hasta finales de 2012, de manera más pronunciada en el sur-sureste (1.9\%) y en el norte (1.2\%) que en el centro $(0.8 \%$, notablemente menor) (cuadro 5). De esta manera, el aumento de las tasas de pobreza considerando ambas fases (II y III) resultó mayor en las entidades del sur-sureste del país $(4.3 \%)$, que en las del centro (4\%) y que en las del norte

${ }^{21}$ Los coeficientes de correlación de Spearman entre la evolución trimestral de la TPA de las tres macrorregiones resultaron positivos y altamente significativos $(p=0.01)$ : entre el norte y el centro $(r=0.985)$; entre el norte y el sur-sureste $(r=0.932)$ y entre el centro y y el sur-sureste $(r=0.916)$. Los coeficientes de correlación en términos incrementales son igualmente positivos y estadísticamente significativos $(0.878,0.881$ y 0.867 , respectivamente). 
Cuadro 5. México. TPA estimada trimestralmente ${ }^{1}$ por entidad federativa y agregada por macrorregión al inicio y final del ciclo económico, y variación, 2005-2012 (porcentajes de la población nacional)

\begin{tabular}{|c|c|c|c|c|c|c|c|c|c|c|}
\hline \multirow{2}{*}{$\begin{array}{c}\text { Macrorregiones } \\
\text { y entidades } \\
\text { federativas }\end{array}$} & \multicolumn{3}{|c|}{ Fase I: Expansión } & \multicolumn{3}{|c|}{ Fase II: Contracción } & \multicolumn{3}{|c|}{ Fase III: Recuperación } & \multirow{2}{*}{$\begin{array}{l}\text { Fase } \\
\text { II y III } \\
\text { Delta }{ }^{3}\end{array}$} \\
\hline & $2005_{1}$ & $2007_{4}$ & Delta $^{2}$ & $2008_{1}$ & $2009_{4}$ & Delta ${ }^{2}$ & $2010_{1}$ & $2012_{4}$ & Delta $^{2}$ & \\
\hline Norte & 8.6 & 7.9 & -0.7 & 8.3 & 10.3 & 2.4 & 10.3 & 11.5 & 1.2 & 3.6 \\
\hline Baja California & 1.3 & 1.3 & 0.0 & 1.4 & 2.3 & 1.0 & 2.1 & 2.5 & 0.2 & 1.2 \\
\hline $\begin{array}{l}\text { Baja California } \\
\text { Sur }\end{array}$ & 4.7 & 3.6 & -1.1 & 4.3 & 6.9 & 3.2 & 5.8 & 6.0 & -0.8 & 2.4 \\
\hline Coahuila & 8.6 & 7.9 & -0.7 & 9.2 & 11.1 & 3.2 & 11.6 & 10.7 & -0.4 & 2.7 \\
\hline Chihuahua & 8.6 & 7.2 & -1.3 & 7.3 & 10.4 & 3.2 & 10.6 & 10.5 & 0.1 & 3.2 \\
\hline Nayarit & 17.2 & 14.8 & -2.4 & 16.1 & 17.8 & 3.0 & 17.4 & 19.2 & 1.4 & 4.4 \\
\hline Nuevo León & 3.6 & 3.8 & 0.2 & 4.5 & 6.0 & 2.1 & 5.9 & 7.6 & 1.7 & 3.8 \\
\hline Sinaloa & 13.7 & 13.8 & 0.1 & 13.6 & 15.0 & 1.2 & 14.8 & 18.2 & 3.2 & 4.4 \\
\hline Sonora & 9.6 & 9.2 & -0.4 & 9.7 & 11.3 & 2.2 & 12.9 & 14.1 & 2.8 & 4.9 \\
\hline Tamaulipas & 10.3 & 9.3 & -1.0 & 8.4 & 11.8 & 2.5 & 12.0 & 14.7 & 2.9 & 5.4 \\
\hline Centro & 18.7 & 17.9 & -0.8 & 18.8 & 21.1 & 3.2 & 20.6 & 21.9 & 0.8 & 4.0 \\
\hline Aguascalientes & 14.9 & 14.8 & $\begin{array}{ll}-0.1 \\
\end{array}$ & 15.5 & 18.6 & 3.9 & 18.2 & 19.6 & 1.0 & 4.8 \\
\hline Colima & 8.9 & 7.2 & -1.7 & 8.7 & 10.5 & 3.3 & 9.3 & 10.3 & -0.3 & 3.1 \\
\hline Distrito Federal & 5.4 & 6.3 & 0.8 & 6.4 & 8.1 & 1.8 & 7.3 & 8.8 & 0.8 & 2.6 \\
\hline Durango & 24.4 & 20.4 & -4.0 & 21.6 & 26.5 & 6.0 & 24.8 & 24.8 & -1.7 & 4.4 \\
\hline $\begin{array}{l}\text { Estado de } \\
\text { México }\end{array}$ & 14.3 & 14.5 & 0.2 & 15.0 & 17.8 & 3.3 & 16.6 & 19.3 & 1.5 & 4.8 \\
\hline Guanajuato & 18.9 & 19.4 & 0.5 & 21.4 & 24.6 & 5.2 & 24.8 & 25.5 & 1.0 & 6.1 \\
\hline Hidalgo & 25.7 & 25.8 & 0.2 & 27.5 & 28.8 & 3.0 & 29.3 & 28.0 & -0.8 & 2.2 \\
\hline Jalisco & 10.9 & 9.9 & -1.0 & 10.1 & 12.6 & 2.6 & 11.4 & 11.2 & -1.4 & 1.3 \\
\hline Michoacán & 23.3 & 22.5 & -0.8 & 22.4 & 22.1 & -0.4 & 24.3 & 27.1 & 5.0 & 4.7 \\
\hline Morelos & 10.7 & 10.4 & -0.3 & 10.8 & 12.5 & 2.2 & 11.8 & 12.9 & 0.4 & 2.5 \\
\hline Puebla & 26.7 & 26.1 & -0.6 & 27.4 & 29.4 & 3.4 & 29.6 & 30.3 & 0.9 & 4.3 \\
\hline Querétaro & 12.5 & 12.0 & -0.5 & 12.5 & 14.3 & 2.4 & 13.6 & 15.8 & 1.5 & 3.9 \\
\hline San Luis Potosí & 25.7 & 25.8 & 0.1 & 26.6 & 29.5 & 3.7 & 29.3 & 32.2 & 2.7 & 6.4 \\
\hline Tabasco & 28.5 & 23.4 & -5.1 & 24.6 & 27.9 & 4.5 & 26.9 & 32.1 & 4.2 & 8.7 \\
\hline Tlaxcala & 17.9 & 19.6 & 1.7 & 21.2 & 25.3 & 5.7 & 24.4 & 24.1 & -1.3 & 4.5 \\
\hline Veracruz & 28.0 & 27.1 & -0.9 & 26.7 & 28.8 & 1.7 & 28.6 & 28.7 & -0.1 & 1.6 \\
\hline Zacatecas & 20.9 & 19.0 & -1.9 & 20.5 & 20.9 & 1.9 & 20.5 & 21.9 & 1.0 & 2.9 \\
\hline Sur-sureste & 29.4 & 28.7 & -0.6 & 28.8 & 31.1 & 2.3 & 30.8 & 33.0 & 1.9 & 4.3 \\
\hline Campeche & 20.0 & 17.8 & -2.2 & 17.8 & 18.4 & 0.6 & 19.3 & 19.0 & 0.6 & 2.2 \\
\hline Chiapas & 47.0 & 45.3 & -1.7 & 44.8 & 45.9 & 0.6 & 45.0 & 47.4 & 1.4 & 2.1 \\
\hline Guerrero & 42.0 & 41.8 & -0.2 & 42.0 & 45.3 & 3.5 & 44.9 & 51.1 & 5.7 & 9.2 \\
\hline Quintana Roo & 11.0 & 11.3 & 0.3 & 10.7 & 14.2 & 2.9 & 14.3 & 16.3 & 2.1 & 5.1 \\
\hline Oaxaca & 38.1 & 39.2 & 1.0 & 40.1 & 43.4 & 4.2 & 42.2 & 43.8 & 0.4 & 4.6 \\
\hline Yucatán & 18.1 & 17.0 & -1.1 & 17.3 & 19.2 & 2.2 & 19.0 & 20.3 & 1.1 & 3.3 \\
\hline Nacional & 18.2 & 17.8 & -0.4 & 18.4 & 21.3 & 3.5 & 20.7 & 22.6 & 1.3 & 4.8 \\
\hline
\end{tabular}

${ }^{1}$ Porcentaje de la población en condiciones de pobreza alimentaria en el año y trimestre que se indica.

${ }^{2}$ Aumento (disminución) entre el final de una fase y el inicio de la otra (puntos porcentuales).

${ }^{3}$ Aumento (disminución) de la TPA durante las fases I y III de manera agregada (puntos porcentuales).

Fuente: Estimaciones propias a partir de información de CONEVAL, procesada con la metodología explicada en el texto. 
Gráfica 3. México. Evolución trimestral de la TPA estimada para tres macrorregiones, $2005_{1}$ a $2012_{4}$

(porcentaje de la población)

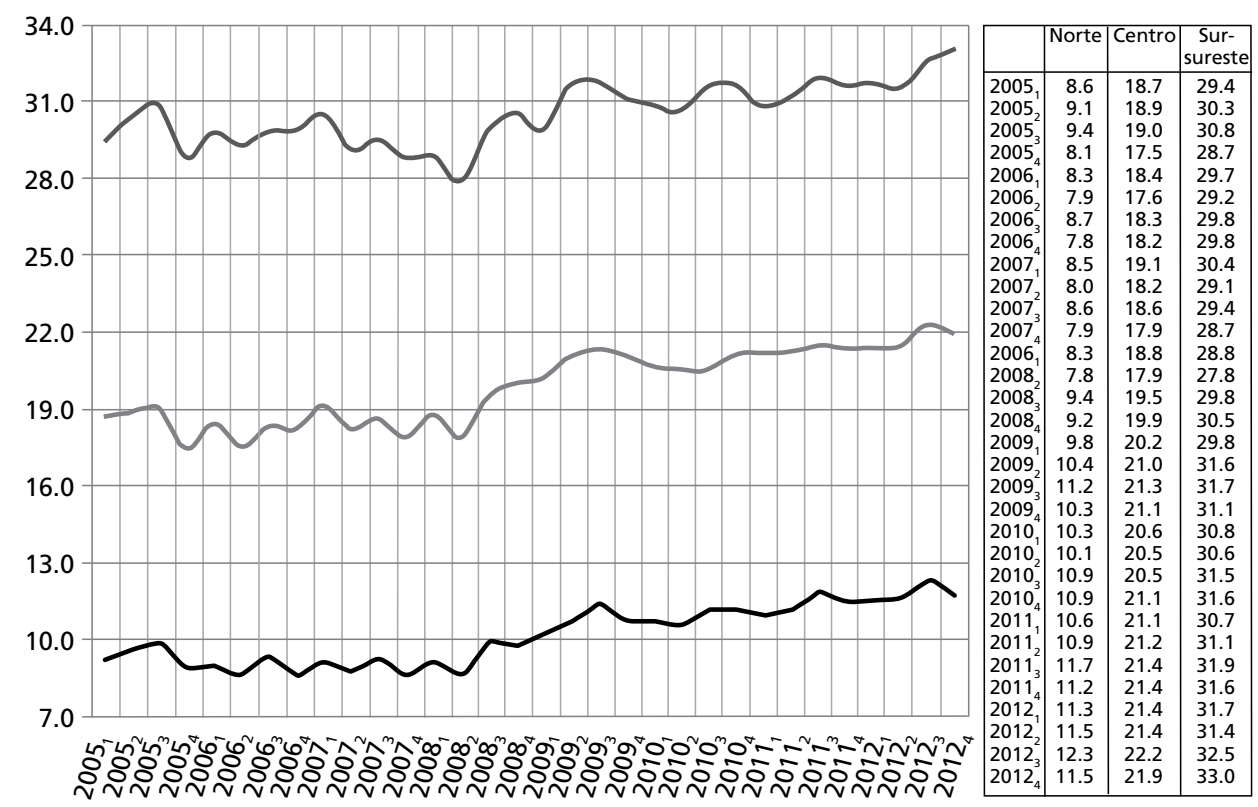

Norte

Centro

Sur-sureste

Fuente: Cálculos propios con la metodología y agregación descritos en el texto.

(3.6\%), aunque no cabría descartar que estas tendencias estuviesen influidas de alguna manera por los sesgos en las estimaciones señalados más arriba.

Aunque el sur-sureste no resultó muy afectado en la fase recesiva, sí lo fue durante la fase de recuperación, de manera que tomadas en conjunto se vieron especialmente perjudicadas entidades como Guerrero (9.2\%) y Quintana Roo (5.1\%), por la aguda contracción del turismo internacional; en contraste, otros estados de esa misma macrorregión vinculados con sectores económicos tradicionales, como Yucatán, Chiapas y Campeche, experimentaron una contracción menos severa (por debajo de 3.5\%). En el centro, el aumento de la TPA fue también de significación en las dos últimas fases del ciclo, tanto en entidades tradicionalmente rezagadas, como Tabasco y San Luis Potosí (más de 6\%), como en otras de nueva industrialización como Guanajuato, Aguascalientes, Querétaro y Puebla (entre 4\% y 6\%). Por último, algunos estados del norte vieron crecer, también de manera singular, su pobreza alimentaria en ambas etapas, especialmente las fronterizas que alojan los principales centros maquiladores de exportación del 
país, como Tamaulipas y Sonora (más de 5\%), y menos severamente Nuevo León, Coahuila, Baja California y Baja California Sur (cuadro 5).

En resumen, lo generalizado de la contracción y de la lenta recuperación a lo largo y ancho del territorio nacional sugiere que la propagación regional de la crisis, aunque de manera diferenciada en cada macrorregión, fue especialmente severa en las entidades abocadas al turismo y a las manufacturas de exportación del centro y del norte del país, en comparación con los estados preferentemente orientados al mercado interno, en especial los más vinculados con sectores económicos tradicionales tanto del centro como del sur-sureste.

\section{EL MERCADO LABORAL Y LOS FACTORES DETERMINANTES DE LA POBREZA: UN ANÁLISIS CUANTITATIVO}

Diversos estudios analíticos que abordan el examen cuantitativo de la relación entre el ciclo económico y la pobreza destacan preferentemente los efectos de algunos instrumentos de política pública utilizados para mitigarla mediante procesos de ajuste (Demery y Squire,1996; Romer y Romer, 1998). Nuestro interés es, quizás, más modesto, en la medida en que buscamos analizar la relación cuantitativa que se observa entre la evolución del ciclo económico en México y sus efectos sobre la pobreza alimentaria, medida por el desempeño de dos indicadores del mercado laboral. Con ese objetivo, en este apartado tratamos de explicar la evolución trimestral de la TDA de las 32 entidades federativas del país, a lo largo de los 32 trimestres que duró el ciclo que va desde los antecedentes hasta las secuelas que dejó en la economía mexicana la gran recesión global de 2008 y 2009.

Para ello, inicialmente planteamos dos hipótesis: 1) dado el carácter contracíclico que adopta la TDA en la mayoría de los países, esperaríamos que en México la pobreza alimentaria se incrementara en la fase recesiva y tendiera a aminorarse -aunque de manera más pausada- durante la recuperación, especialmente en el caso de crisis financieras como la recientemente registrada; 2) por otra parte, y aunque no existe consenso entre los especialistas, ${ }^{22}$ la evidencia sobre México indica un doble comportamiento (pro- y contracíclico) del em-

\footnotetext{
${ }^{22}$ En efecto, suele aceptarse que en la medida en que la ocupación informal resulta de segmentaciones del mercado laboral, su comportamiento es típicamente contracíclico (Loayza y Rigolini, 2006), en tanto que si no existen segmentaciones significativas, se sostiene que su carácter es procíclico, es decir, aumenta el autoempleo informal en las fases expansivas del ciclo y disminuye en las recesiones (Boeri y Van Ours, 2008). Un extenso examen de países latinoamericanos concluye que en los mercados laborales de los países de la región se comprueba la presencia de mayor
} 
pleo informal a lo largo del ciclo económico completo (Maloney, 1998; Loayza y Sugawara, 2009; Hernández Laos, Solís y Stefanovich, 2013), por lo que no establecemos a priori el sentido de la causalidad entre la evolución de la actividad económica durante el ciclo y sus efectos sobre la tasa de empleo informal. Con base en lo anterior, en caso de resultar contracíclico el comportamiento conjunto de ambos indicadores, en la segunda parte del ejercicio esperaríamos que en la fase contractiva se redujeran los ingresos de los hogares afectados, lo que aumentaría la TPA. Ello tendería a revertirse durante la recuperación de la economía, aunque posiblemente con escaso dinamismo en el mediano plazo, si se rezaga la recuperación del empleo formal y de los ingresos reales.

La cuantificación se lleva a cabo mediante dos modelos dinámicos de regresión con un panel de datos que cubre las entidades federativas $(i=1 \ldots 32)$ y los 32 trimestres que abarcaron las tres fases de este ciclo económico $(t=$ $2005_{1} \ldots 2012_{4}$ ). Primeramente relacionamos los indicadores del mercado laboral con las variaciones estatales y trimestrales de la actividad económica sectorial (modelo I); después cuantificamos los efectos ejercidos por los indicadores laborales sobre la TDA de cada entidad (modelo II).

De acuerdo con otros ejercicios empíricos similares ${ }^{23}$ el modelo I adopta una especificación que permite cuantificar de manera dinámica los efectos del comportamiento sectorial de la economía sobre los dos indicadores ya examinados del mercado laboral, la TDA y la TOSI, como se expresa a continuación:

$$
\begin{aligned}
& \ln \left(T D A_{i, t}\right)=\rho_{1} \ln \left(T D A_{i, t-1}\right)+\Sigma \beta_{1}^{k} \ln \left(I E_{i, t}^{k}\right)+\lambda_{1, t}+\mu_{1, i}+\varphi_{1, i, t}+\epsilon_{i t} \\
& \ln \left(\operatorname{TOSI}_{i, t}\right)=\rho_{2} \ln \left(\operatorname{TOSI}_{i, t-1}\right)+\Sigma \beta_{2}^{k} \ln \left(I E_{i, t}^{k}\right)+\lambda_{2 t}+\mu_{2 i}+\varphi_{2, i, t}+\epsilon_{i t}
\end{aligned}
$$

donde los subíndices $i$ y $t$ expresan, respectivamente, las entidades federativas $(i=1 \ldots 32)$ y los trimestres analizados $\left(t=2005_{1} \ldots 2012_{4}\right)$. Se procesa la información con un panel dinámico, introduciendo como explicativas la variable dependiente con un rezago trimestral, y se introducen $k$ indicadores económicos sectoriales $(k=1 \ldots 3)$ de los estados, cuya evolución afecta el comportamiento de los indicadores laborales; $\lambda_{t}$, representa los efectos temporales agregados; $\mu_{i}$ у $\phi_{i t}$,

segmentación durante las recesiones y mayor integración en las recuperaciones, lo que explica su doble carácter pro- y contracíclico en ambas fases (Perry et al., 2008).

${ }^{23}$ Los esquemas empíricos que aplicamos parten del modelo utilizado por Gundersen y Ziliak (2004) para cuantificar los determinantes de la evolución de la pobreza en los cincuenta estados de Estados Unidos. 


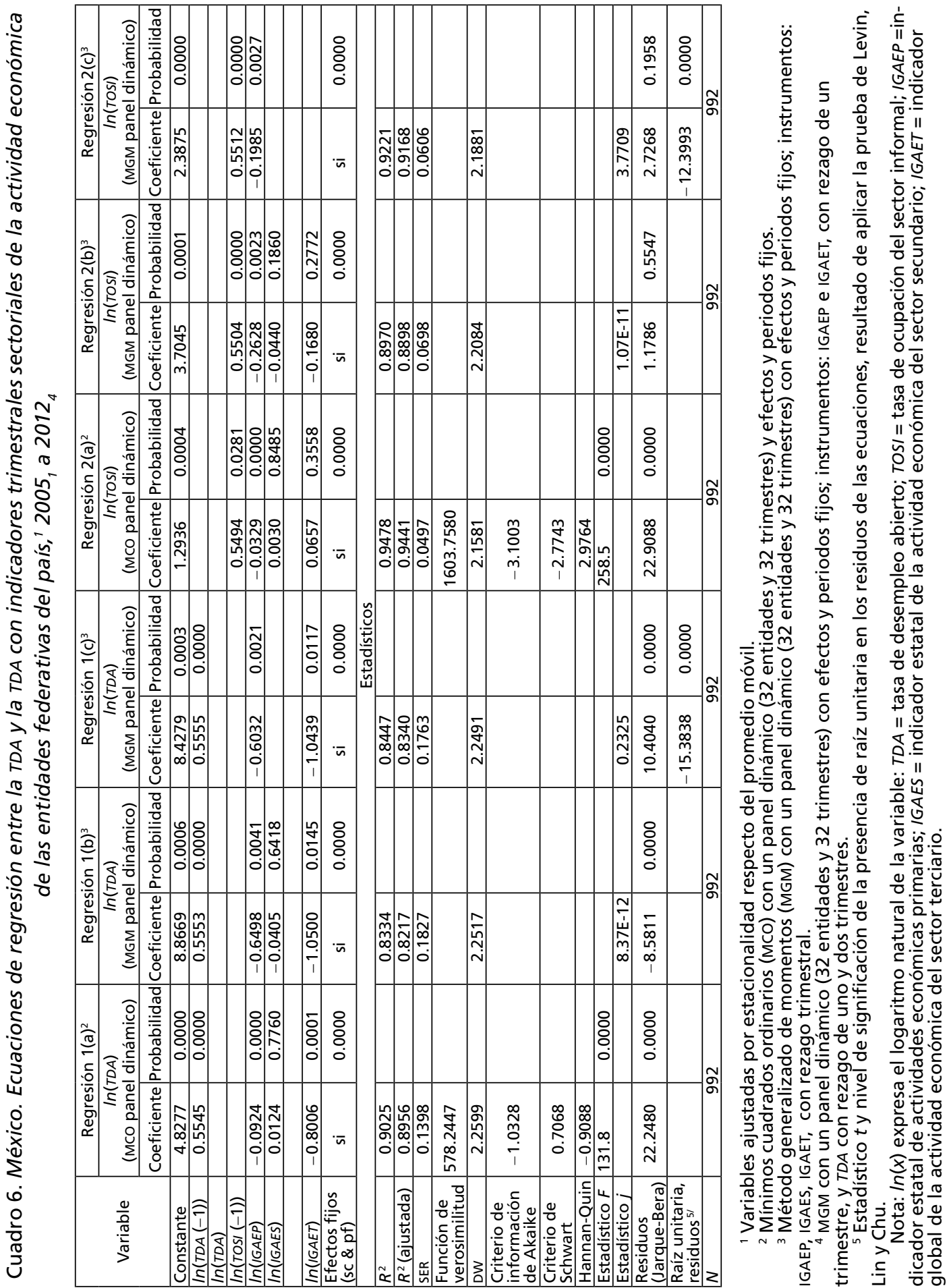


los efectos fijos y de tendencia que son específicos para cada estado, y $\epsilon_{i t}$, un error aleatorio.

En ambas ecuaciones incluimos un rezago trimestral de la variable dependiente porque es posible esperar, a nivel agregado de las entidades, que ambos indicadores laborales (en especial el empleo informal) respondan con relativa lentitud a los cambios trimestrales de las condiciones económicas. Como indicador de la evolución trimestral utilizamos el Indicador General de la Actividad Económica Estatal, con una desagregación sectorial para identificar los efectos diferenciados de los sectores primario (IGAEP), secundario (IGAES) y terciario (IGAET) sobre los indicadores del mercado laboral. ${ }^{24}$

El cuadro 6 despliega diferentes estimaciones de ambas ecuaciones. Las regresiones 1(a) y 2(a) son estimaciones dinámicas por mínimos cuadrados ordinarios (MCO) con efectos temporales y fijos, ${ }^{25}$ las cuales cumplen las pruebas estadísticas usuales en este tipo de regresiones. ${ }^{26}$ Como era de esperarse, ambas estimaciones muestran que tanto TDA como TOSI se ven afectadas por sus propios niveles del trimestre previo y que los coeficientes de determinación de las estimaciones son elevados y estadísticamente significativos. Sin embargo, se detecta que en tanto la TDA registra una elasticidad negativa y estadísticamente significativa respecto del IGAEP y del IGAET, la TOSI sólo muestra elasticidad negativa y estadísticamente significativa respecto del IGAEP, pero no respecto del IGAES y el IGAET.

Los resultados anteriores, si bien son de interés, podrían estar afectados en algún grado por la endogeneidad de las variables independientes $;{ }^{27}$ para evitar este problema se recurrió al despeje de ambas ecuaciones por el método generalizado de momentos (MGM), utilizando como instrumentos las mismas variables

\footnotetext{
${ }^{24}$ Las variables fueron ajustadas por estacionalidad respecto de la evolución de su media móvil, y comprobada su raíz unitaria con la prueba de Levin, Lin y Chu, como se muestra en el cuadro A.1 del anexo.

${ }^{25} \mathrm{La}$ introducción de efectos fijos en las estimaciones permite considerar que si bien el efecto de la actividad económica (total o sectorial) es igual para todas las entidades federativas, la constante para cada una de ellas puede ser diferente, lo que estaría reflejando características idiosincráticas de las diversas entidades, derivadas de factores no incluidos en las ecuaciones de regresión. Los efectos fijos temporales permiten identificar las repercusiones en cada entidad federativa que varían trimestralmente en el período de análisis.

${ }^{26}$ Las pruebas estadísticas aplicadas a las cuatro regresiones $(p=0.01)$ fueron: normalidad, exogeneidad y homocedasticidad de los residuos; por falta de espacio no presentamos los resultados de las pruebas ni los valores de los coeficientes de los efectos fijos y temporales.

${ }^{27}$ Como lo confirma el análisis de causalidad de Granger entre algunas de las variables dependientes y las dos independientes.
} 
pero con un rezago trimestral para garantizar una mayor exogeneidad, como se muestra en las regresiones 1(b) y 2(b) del mismo cuadro 6. Estas nuevas estimaciones confirman las anteriores respecto al signo y la significación de las elasticidades respectivas, pero ilustra con claridad que los efectos de la economía sobre los indicadores laborales son mayores en términos absolutos que los mostrados en las estimaciones anteriores. Dada la escasa significación estadística de los efectos del IGAES sobre la TDA, la regresión 1(c), realizada también por el MGM con las mismas variables instrumentales, describe los efectos estadísticamente significativos sobre la TDA y cumple con las diferentes pruebas estadísticas usuales, cuyos indicadores se muestran en la parte inferior del mismo cuadro. Estos resultados confirman con claridad los efectos contracíclicos que imponen las actividades primarias y terciarias sobre el desempleo abierto, siendo de mayor relevancia en términos absolutos los del sector terciario que los del primario.

En el caso de la regresión 2(b), el cálculo por MGM, utilizando como instrumentos los mismos indicadores de la actividad sectorial con un rezago trimestral, pone de manifiesto, a juzgar por el signo negativo de los parámetros correspondientes, el efecto contracíclico que ejerce la actividad económica sobre la Tosi; sin embargo, sólo la del sector primario tiene significación estadística $(p=0.01)$, no así las secundarias y terciarias. Para cuantificar sólo los efectos estadísticamente significativos, la regresión 2(c) muestra los valores esperados de los parámetros, lo que confirma el carácter contracíclico del empleo informal generado por los movimientos en las actividades primarias.

Examinemos ahora el modelo II, que cuantifica los efectos del mercado laboral (TDA y TDA) sobre la TPA de las entidades federativas. En este caso se adopta una especificación similar a la propuesta por Gurdensen y Ziliak (2006, pp. 66-67) que también implica medias condicionadas lineales:

$$
\ln \left(T P A_{i, t}\right)=p^{\prime}{ }_{1} \ln \left(T P A_{i, t-1}\right)+\Sigma \beta^{\prime k} \ln \left(I M L_{i, t}^{k}\right)+\lambda_{1, t}^{\prime}+\mu_{1, i}^{\prime}+\varphi_{1, i, t}^{\prime}+\epsilon_{i t}^{\prime}
$$

Como en el anterior, en este modelo las variables también se ajustan por estacionalidad respecto a su media móvil y se comprueba que son estacionarias de grado uno. ${ }^{28}$ En esta ecuación, los subíndices $i$ y $t$ expresan, respectivamente, las entidades federativas $(i=1 \ldots 32)$ y los trimestres analizados $\left(t=2005_{1} \ldots 2012_{4}\right)$;

\footnotetext{
${ }^{28}$ Se aplicó la prueba de raíz unitaria de panel, utilizando el kernel de Bartlett, y se obtuvieron valores del estadístico $t$ (prueba de Levin, Lin y Chu) que resultan significativos estadísticamente $(p=0.01)$; por razones de espacio no se presentan los resultados.
} 
la información se procesa de manera dinámica, introduciendo como explicativa la variable dependiente con un rezago trimestral, y se incluyen $k$ indicadores del desempeño del mercado laboral $\left(I M L^{k}, k=1,2\right)$, cuya evolución afecta el comportamiento de la TPA; $\lambda^{\prime}$, expresa los efectos temporales agregados de esta relación; $\mu_{i}^{\prime}{ }_{i} \varphi^{\prime}{ }_{i t}$, los efectos fijos y de tendencia específicos de los estados, y $\epsilon_{i t}^{\prime}$ es un error aleatorio.

En (3) se incluye un rezago trimestral en la variable dependiente porque, al persistir en los hogares, a nivel agregado de las entidades las tasas de pobreza responden lentamente a los cambios trimestrales de las condiciones económicas (Gundersen y Ziliak, 2004, p. 68). En las especificaciones 3(a) y 3(b) introducimos la variable de la TOSI con su valor corriente $\left(\operatorname{TOSI}_{i, t}\right)$ y en la $(3 \mathrm{c})$ con un rezago trimestral $\left(\operatorname{TOSI}_{i, t-1}\right)$, porque resulta razonable suponer que sus efectos sobre la pobreza demoren en presentarse, por ejemplo, un trimestre, en tanto que las repercusiones de la TDA se manifiesten de manera más inmediata, es decir, en el mismo trimestre en que se ve perturbada la actividad económica. ${ }^{29}$

El cuadro 7 muestra los resultados de las regresiones. La 3(a) se calcula por MCO, en tanto que en la 3(b) y la 3(c) se aplica el MGM, las cuales pasan satisfactoriamente las pruebas estadísticas usuales..$^{30}$ Las tres regresiones muestran, por una parte, la demora trimestral que toma el ajuste de la TPA corriente a cambios en las condiciones del mercado laboral del trimestre previo, lo que confirma lo adecuado del enfoque dinámico aplicado. Si bien se toma como variables explicativas los dos indicadores del desempeño laboral, en las primeras dos (3a y $3 b$ ) se supone que sus efectos tienen lugar en el trimestre corriente, en tanto que para la (3c) se asume, por las razones apuntadas más arriba, que la Tosi tarda un trimestre en agotar sus efectos sobre la tasa de pobreza de las entidades federativas, por lo que se especifica con un rezago trimestral $\left(\right.$ TOSI $\left._{t-1}\right)$.

\footnotetext{
${ }^{29}$ La razón del rezago se basa en la posibilidad de que parte importante de los que ingresan a las ocupaciones informales en la etapa recesiva estuvieron un tiempo (un trimestre, por ejemplo) en condiciones de desempleo abierto, a la espera de obtener de nuevo un trabajo formal; al no conseguirlo, y dada la ausencia de un seguro de desempleo universal en México, se podría esperar que, conforme se profundiza la contracción y por la necesidad de percibir algún ingreso, una proporción de esas personas se inserta en ocupaciones informales, lo que puede colocar a sus hogares en condiciones de pobreza alimentaria. De ser así, el proceso se revertiría -al menos parcialmente- en la etapa de recuperación, confirmando el carácter contracíclico de la informalidad.

${ }^{30}$ En este caso, las pruebas de inferencia estadística aplicadas permiten comprobar también ( $p=0.01)$ la normalidad de los residuos, la ausencia de autocorrelación y de multicolinealidad entre las variables independientes, y la exogeneidad de las variables explicativas; por falta de espacio tampoco presentamos los resultados de estas pruebas ni los valores de los efectos fijos y temporales de las estimaciones.
} 
88 ECONOMÍA: TEORÍA Y PRÁCTICA • Nueva Época, número 40, enero-junio 2014

Cuadro 7. México. Ecuaciones de regresión entre la TPA con la TDA y la TOSI de las 32 entidades federativas del país, 2005, a $2012_{4}{ }^{1}$

\begin{tabular}{|c|c|c|c|c|c|c|}
\hline \multirow[t]{2}{*}{ Variable } & \multicolumn{2}{|c|}{$\begin{array}{c}\text { Regresión 3(a)² } \\
\text { In(TPA) } \\
\text { (MCO - panel dinámico) }\end{array}$} & \multicolumn{2}{|c|}{$\begin{array}{c}\text { Regresión } 3(b)^{3} \\
\ln (T P A) \\
\text { (MGM - panel dinámico) }\end{array}$} & \multicolumn{2}{|c|}{$\begin{array}{c}\text { Regresión } 3(\mathrm{c})^{4} \\
\ln (T P A) \\
\text { (MGM - panel dinámico) }\end{array}$} \\
\hline & Coeficiente & Probabilidad & Coeficiente & Probabilidad & Coeficiente & Probabilidad \\
\hline Constante & 0.4015 & 0.0000 & -0.0693 & 0.7006 & 0.3209 & 0.0000 \\
\hline $\operatorname{In}(T P A(-1))$ & 0.7559 & 0.0000 & 0.7549 & 0.0000 & 0.7590 & 0.0000 \\
\hline $\ln (T P A)$ & 0.0712 & 0.0000 & 0.0425 & 0.0372 & 0.0588 & 0.0020 \\
\hline $\ln ($ TOSI) & 0.0544 & 0.0522 & 0.2114 & 0.0003 & & \\
\hline $\ln ($ TOSI $(-1))$ & & & & & 0.0816 & 0.0058 \\
\hline $\begin{array}{l}\text { Efectos fijos } \\
\text { (sc \& PF) }\end{array}$ & si & 0.0000 & si & 0.0000 & si & 0.0000 \\
\hline
\end{tabular}

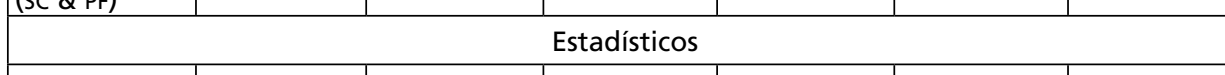

\begin{tabular}{|l|l|l|l|l|l|l|}
\hline$R^{2}$ & 0.9954 & & 0.9952 & & 0.9954 & \\
\hline$R^{2}$ (ajustada) & 0.9950 & & 0.9949 & & 0.9950 & \\
\hline SER & 0.0489 & & 0.0497 & & 0.0489 & \\
\hline Funcion & & & & & \\
\hline
\end{tabular}

\begin{tabular}{|l|c|l|c|l|l|l|}
\hline $\begin{array}{l}\text { Función de } \\
\text { verosimilitud }\end{array}$ & 1619.79 & & & & & \\
\hline DW & 2.4928 & & 2.4631 & & 2.4826 & \\
\hline Criterio de & & & & & \\
\hline
\end{tabular}

\begin{tabular}{|c|c|c|c|c|c|c|}
\hline $\begin{array}{l}\text { Criterio de } \\
\text { información } \\
\text { de Akaike }\end{array}$ & -3.1347 & & & & & \\
\hline $\begin{array}{l}\text { Criterio de } \\
\text { Schwart }\end{array}$ & -2.8136 & & & & & \\
\hline Hannan-Quin & -3.0126 & & & & & \\
\hline Estadístico $F$ & 3104.8 & 0.0000 & & & & \\
\hline Estadístico $j$ & & & 22.7294 & & 29.9501 & \\
\hline $\begin{array}{l}\text { Residuos } \\
\text { (Jarque-Bera) }\end{array}$ & 22.9954 & 0.0000 & 28.2223 & 0.0000 & 26.1754 & 0.0000 \\
\hline $\begin{array}{l}\text { Raíz unitaria, } \\
\text { residuos }^{5}\end{array}$ & -0.1238 & 0.0000 & -12.0815 & 0.0000 & -11.5070 & 0.0000 \\
\hline$N$ & \multicolumn{2}{|c|}{992} & \multicolumn{2}{|c|}{992} & \multicolumn{2}{|c|}{992} \\
\hline
\end{tabular}

${ }^{1}$ Variables ajustadas por estacionalidad respecto del promedio móvil.

2 Mínimos cuadrados ordinarios (MCO) con un panel dinámico (32 entidades y 32 trimestres) y efectos y periodos fijos.

${ }^{3}$ Método generalizado de momentos (MGM) con un panel dinámico (32 entidades y 32 trimestres) con efectos y periodos fijos; instrumentos: índices de la actividad económica estatal con rezago de un trimestre, al igual que la TDA y LA TOSI.

${ }^{4}$ MGM con un panel dinámico (32 entidades y 32 trimestres) con efectos y periodos fijos; instrumentos: índices de la actividad económica estatal y TDA con rezago de un trimestre, y TOS। con rezago de dos.

Nota: $\ln (x)$ expresa el logaritmo natural de la variable: TPA = tasa de pobreza alimentaria; $T D A=$ tasa de desempleo abierto; TOSI = tasa de ocupación del sector informal. 
Como se observa en el cuadro 7, los resultados de la regresión 3(a) muestran que las elasticidades de la TDA y de la TOSI sobre la TPA son positivas, aunque sólo es estadísticamente significativa la primera $(p=0.01)$, no así la segunda $(p<0.01)$. En el cálculo por MGM (regresión $3 \mathrm{~b}),{ }^{31}$ si bien no se modifica el signo de las elasticidades, sí cambia significativamente su valor absoluto, lo que podría indicar la presencia de endogeneidad en las estimaciones anteriores. En 3(b) se detecta que la elasticidad de la TPA respecto de cambios en la TOSI es del orden de cinco veces que la de la TDA, aunque en este cálculo la primera no alcanza el nivel de significación estadística requerido.

Por lo anterior, y dada la ligera correlación entre TDA y TOSI, ${ }^{32}$ optamos por introducir la TOSI con un rezago trimestral, como se muestra en la regresión 3(c). Esta ecuación, que pasa todas las pruebas estadísticas usuales, muestra con un elevado grado de significación estadística $(p=0.01)$ que la elasticidad de la TDA sobre la TPA es relativamente mayor $(\approx+0.06)$, lo que indica que, dado el carácter contracíclico de la TDA, sus movimientos afectan de manera directa y estadísticamente significativa la TPA, acrecentándola en la etapa recesiva y disminuyéndola durante la recuperación. En contraste, las variaciones de la informalidad, a pesar de su carácter también contracíclico, parecieran no incidir de manera significativa sobre los indicadores de pobreza alimentaria en el mismo periodo en que ocurren, sino que su efecto se presenta un trimestre después de que se ve perturbada la actividad económica, con una elasticidad cuyo valor absoluto es ligeramente mayor al que registran las variaciones del desempleo abierto en el período corriente $(\approx 0.8)$.

A partir de los planteamientos conceptuales expuestos y de los resultados obtenidos en las estimaciones econométricas descritas por los dos modelos planteados, y tomando sólo los resultados más consistentes y confiables (regresiones $1 \mathrm{c}, 2 \mathrm{c}$ y $3 \mathrm{c})$, en términos estadísticamente significativos $(p=0.01)$ podemos inferir lo siguiente:

1. Las perturbaciones de la actividad primaria y de la terciaria, provocadas por el ciclo económico, afectan procíclicamente a la TDA, y las de las actividades primarias a las tasas de empleo informal de las entidades federativas, acrecentándolas durante la fase recesiva y reduciéndolas en

\footnotetext{
${ }^{31}$ Para el que se toman como instrumentos los dos indicadores laborales y los indicadores económicos sectoriales con un rezago trimestral.

${ }^{32}$ El coeficiente de correlación entre ambas variables $(r=-0.0801)$ con 1024 observaciones es $\neq 0 ; p=0.05)$.
} 
la recuperación, al contrario de las variaciones de la actividad secundaria, que parecieran no repercutir de manera estadísticamente significativa sobre ninguno de los dos indicadores del mercado laboral mexicano.

2. La contracción de las actividades primarias y de las terciarias acrecentó la desocupación abierta de manera más acentuada que la informalidad y, a la vez, las repercusiones del sector terciario sobre la TDA son mayores que las del primario.

3. En consecuencia, además del encarecimiento de la canasta alimentaria durante la gran recesión de 2008-2009, las perturbaciones de la actividad económica repercutieron desfavorablemente sobre el mercado laboral, lo que afectó aún más las condiciones de bienestar de los hogares mexicanos al acrecentar la incidencia de la pobreza alimentaria en los hogares en los que uno o más de sus miembros perdieron el empleo durante la etapa recesiva y/o en aquellos que, con un rezago trimestral, se insertaron en la informalidad con objeto de compensar, al menos parcialmente, los ingresos perdidos.

4. Por último, y en términos cuantitativos, destaca el que los efectos desfavorables del ciclo económico sobre la pobreza alimentaria provocados por el desempleo son estadísticamente menores en valor absoluto $(|\eta| \approx 0.06)$ que los inducidos un trimestre después de haber incidido sobre la ocupación informal $(|\eta| \approx 0.08)$.

Sin duda, los resultados anteriores corroboran que la asociación entre el ciclo económico y la pobreza alimentaria estuvo mediada en parte importante por el desempeño del mercado laboral. En términos esquemáticos, es posible afirmar, entonces, que la recesión de 2008-2009, por su origen externo, al contraer las exportaciones manufactureras y repercutir, además, desfavorablemente sobre las actividades terciarias, acrecentó la TPA de los estados norteños del país, principalmente como consecuencia del aumento en sus niveles de desempleo abierto. En las entidades centrales, junto con el sector secundario, la recesión afectó también a las actividades primarias y terciarias, aumentando no sólo el desempleo, sino la tasa de informalidad que, con un rezago trimestral, habría tendido a incrementar la pobreza alimentaria de los hogares. Por último, en los estados del sur-sureste, en los que predominan las estructuras tradicionales de producción, los efectos de la contracción sobre las actividades económicas en general fueron menos acentuados y, en parte por ello, las repercusiones sobre la pobreza alimentaria fueron notablemente menores. 


\section{EPÍLOGO: CONVERGENCIA REGIONAL DE LA TPA}

La relación observada entre el ciclo económico y la pobreza alimentaria tuvo repercusiones muy interesantes sobre la evolución de la TPA de las entidades federativas, cuyos valores particulares tendieron a converger durante la fase recesiva en términos absolutos. A partir de la hipótesis de Gerschenkron (1962) y Abramovitz (1986), ${ }^{33}$ Ravallion (2009) enunció las condiciones para la convergencia de la tasa de pobreza entre países (regiones), al señalar que ésta depende de la distribución inicial del ingreso de las regiones atrasadas, ya que una desigualdad muy acentuada puede anular las ventajas del atraso de las economías rezagadas.

En esta investigación se parte de otra hipótesis diferente, aunque relacionada, propuesta por Cortés y Rubalcava (1991), sobre la tendencia a la igualación por empobrecimiento. Esta propuesta, basada en la observación del caso mexicano, sostiene que la desigualdad de la distribución del ingreso en nuestro país disminuye durante las etapas recesivas y se incrementa en las expansivas. La redistribución que suele observarse durante las fases contractivas del ciclo en México se acompaña del empobrecimiento más que proporcional de los hogares ricos con respecto al de los hogares pobres, proceso que se revierte en las etapas expansivas. Las tendencias anteriores se derivan, sostienen dichos autores, de la composición típica de los hogares comprendidos en el decil más rico de la población, formado principalmente por profesionistas, empresarios y burócratas de alto nivel, cuyos ingresos suelen disminuir con la recesión y aumentar con la recuperación de manera más que proporcional que los del resto de los hogares mexicanos.

Extrapolando esa regularidad empírica al ámbito geográfico, observamos que el patrón sectorial-regional de la reciente crisis, al manifestarse con mayor acento en las entidades económicamente más avanzadas, es decir, en las norteñas y centrales, que en las tradicionalmente rezagadas del sur-sureste del país, y a pesar de los sesgos incurridos en nuestras estimaciones de las tasas de pobreza alimentaria, ${ }^{34}$ incrementó más que proporcionalmente la pobreza ali-

\footnotetext{
${ }^{33}$ Gerschenkron y Abramovitz plantearon lo que ahora conocemos como la hipótesis de la convergencia, con la cual sostuvieron que bajo ciertas condiciones los países (regiones) atrasados crecen más aprisa que las economías ricas, lo que con el tiempo tiende a cerrar la brecha entre ambos grupos.

${ }^{34}$ Recordemos que al compararlas con los cálculos oficiales del CONEVAL basados en las cifras de la ENIGH para 2008 y para 2012, nuestras estimaciones tienden a sobreestimar la TPA de las entidades más pobres del país y a subestimar la de las entidades económicamente más avanzadas.
} 
mentaria en las entidades "ricas" que en las "pobres", provocando una clara trayectoria convergente entre ellas durante la fase recesiva del ciclo 2005-2012.

Cuadro 8. México. Regresiones para evaluar la convergencia $\beta$ de las TPA de las 32 entidades federativas, calculadas a partir de la ENIGH y con promedios anuales de estimaciones trimestrales calculados con índices de pobreza laboral anualizados

\begin{tabular}{|c|c|c|c|c|c|}
\hline \multirow{3}{*}{$\begin{array}{l}\text { Variables } \\
\text { independientes }\end{array}$} & \multicolumn{5}{|c|}{$\begin{array}{l}\text { Variable dependiente: tasa de crecimiento medio anual de la TPA } \\
\text { calculada con }\end{array}$} \\
\hline & \multicolumn{4}{|c|}{ Índices trimestrales de pobreza laboral ${ }^{1}$} & \multirow{2}{*}{$\begin{array}{c}\text { ENIGH }^{2} \\
2005-2012\end{array}$} \\
\hline & $2005_{1}-2007_{4}$ & $2007_{4}-2009_{4}$ & $2009_{4}-2012_{4}$ & $2005_{1}-2012_{4}$ & \\
\hline Constante & -0.003 & $0.309^{4}$ & $0.061^{4}$ & $0.108^{4}$ & $0.249^{4}$ \\
\hline ENIGH2005 & & & & & $-0.079 * *$ \\
\hline IPL2005 & -0.0040 & & & & \\
\hline IPL2007 & & $-0.082^{4}$ & & & \\
\hline IPL2009, & & & $-0.015^{4}$ & & \\
\hline IPL2005 & & & & $-0.028^{4}$ & \\
\hline \multicolumn{6}{|c|}{ Estadísticos } \\
\hline$N$ & 32 & 32 & 32 & 32 & 32 \\
\hline$R^{2}$ & 0.013 & 0.766 & 0.144 & 0.615 & 0.691 \\
\hline$R^{2}$ (ajustada) & -0.020 & 0.758 & 0.012 & 0.602 & 0.682 \\
\hline Estadístico $F$ & 0.382 & 98.171 & 5.028 & 47.873 & 67.200 \\
\hline (Probabilidad) & 0.541 & 0.000 & 0.033 & 0.000 & 0.000 \\
\hline $\begin{array}{l}\text { Velocidad de } \\
\text { convergencia }\end{array}$ & ns & 0.122 & 0.016 & 0.032 & 0.115 \\
\hline Años 5 & ns & 8 & 63 & 32 & 9 \\
\hline
\end{tabular}

${ }^{1}$ Variable dependiente: tasa de crecimiento medio anual de la TPA anualizada, calculada mediante índices de pobreza laboral con la metodología explicada en el texto.

${ }^{2}$ Variable dependiente: tasa de crecimiento medio anual de la TPA calculada por el CONEVAL con información de la ENIGH.

Variables independientes: ENIGH05 = tasa de pobreza alimentaria en el año inicial (2005) calculada con la ENIGH; IPL200 $x_{x}=$ tasa de pobreza inicial en un trimestre y año específicos calculada con la metodología explicada en el texto.

${ }^{3}$ Diferente de 0 a $5 \%$ de probabilidad.

${ }^{4}$ Diferente de 0 a $1 \%$ de probabilidad.

${ }^{5}$ Número de años requeridos para eliminar la dispersión de la TPA entre las entidades a la velocidad de convergencia registrada en cada periodo.

$\mathrm{ns}=$ no significativo. 
Dos métodos permiten evaluar las tendencias convergentes de la tasa de pobreza de las entidades federativas: la convergencia $\sigma$ y la convergencia $\beta$ (no condicional). La primera evalúa si la dispersión de la TPA entre las entidades, medida por la desviación estándar de los logaritmos, tendió a decrecer durante la recesión; la segunda se valora mediante una ecuación de regresión que identifica la velocidad de convergencia entre dos momentos en el tiempo y permite estimar el número de años que tomaría el proceso para disminuir esa dispersión, digamos, a un nivel equivalente a cuatro quintas partes del observado en la etapa inicial del ciclo. ${ }^{35}$

Dado que la convergencia $\sigma$ reclama la existencia de la convergencia $\beta$ (Sala-i-Martin, 2000, pp. 197-98), se examinará primero esta última. Como se observa en el cuadro 8 , las estimaciones para el periodo previo a la recesión $\left(2005_{1}\right.$ a $\left.2007_{4}\right)$ no muestran una tendencia convergente de las TPA estatales, dada la nula significación estadística de la regresión y sus parámetros. Por el contrario, en el período recesivo $\left(2008_{1}\right.$ a $\left.2009_{4}\right)$ dicha tasa sí registra una significativa tendencia convergente, a un ritmo de $12.2 \%$ por año en promedio, que de prolongarse por ocho años más eliminaría la dispersión registrada a finales de la primera etapa del ciclo.

Por último, durante la recuperación $\left(2010_{1}\right.$ a $\left.2012_{4}\right)$ la tendencia concurrente de la tasa de pobreza no eliminó, aunque redujo, la significación estadística de las estimaciones, al disminuir la velocidad de convergencia a sólo $1.6 \%$ por año, lo que implica que para eliminar la dispersión se requeriría que el proceso convergente se prolongase por lo menos 63 años más. Si tomamos el ciclo económico completo $\left(2005_{1}\right.$ a $\left.2012_{4}\right)$, la tendencia acumulada acusa también una trayectoria convergente, en un proceso moderadamente dinámico (3.2\% anual en promedio) que necesitaría 32 años para eliminar la dispersión de las TPA estatales observada al inicio del ciclo, en 2005 (cuadro 8). ${ }^{36}$

${ }^{35}$ La convergencia $\beta$ no condicional se calcula mediante la siguiente ecuación de regresión: $T C M A_{i, t}+T=b_{0}+b_{1} \ln \left(T P A_{i, t}\right)+e_{i, t}$, en donde $T C M A_{i, t, t}+T$ denota el ritmo de crecimiento anual de la tasa de pobreza entre los trimestres $t$ y $t+T$ en la entidad $i(i=1 \ldots 32) ; b_{0}$ es una constante, y $b_{1}$ es la pendiente que indica la dirección del proceso (convergente $<=>b_{1}<0$; divergente $<=>b>0$ ), lo que permite calcular la velocidad de convergencia $\beta=-\ln \left(1+T^{*} b_{1}\right)(T-t)$, y $e_{i, t}$ es un error aleatorio con las propiedades estadísticas usuales.

${ }^{36}$ La convergencia de todo el proceso se confirma si se evalúa con las tasas oficiales de pobreza alimentaria estimadas por el CONEVAL - con base en la ENIGH- entre 2005 y 2012. En este caso, la ecuación de convergencia $\beta$, mostrada también en el cuadro 8 , al ser estadísticamente significativa, confirma el movimiento convergente en ese periodo, con un lapso de convergencia de nueve años para eliminar la dispersión entre las TDA estatales, medida con base en las estimaciones oficiales. 
La convergencia $\beta$ permite examinar la convergencia $\sigma$ a lo largo de los 32 trimestres que abarcó el ciclo. La gráfica 4 muestra la trayectoria seguida por la varianza de los logaritmos de las TPA de las entidades federativas: la línea clara representa su evolución trimestral y la línea oscura la tendencia calculada con el algoritmo Hodrick-Prescott. Como se observa, más allá de las fluctuaciones trimestrales, hacia finales de 2007 e inicios de 2008 comenzó a reducirse de manera perceptible la dispersión, justamente al inicio de la etapa recesiva del ciclo, tendencia que continuó con la misma velocidad hasta el último trimestre de 2009, al concluir dicha etapa. Se aprecia, además, que la trayectoria convergente se prolongó, aunque con menor impulso, hasta finales de 2012, es decir, a lo largo de la modesta fase de recuperación de la economía nacional.

En resumen, constatamos la trayectoria convergente seguida por las TPA estatales durante las fases recesiva y de recuperación. Este proceso convergente es compatible con la hipótesis de la igualdad por empobrecimiento de Cortés y Rubalcava, pero en vez de referirse a la convergencia en la distribución del ingreso de los hogares, valora la convergencia de las tasas de pobreza en el contexto geográfico de la economía nacional en recesión. En este marco, al afectar severamente al sector exportador de la economía, la contracción generó movimientos sectoriales diferenciados sobre las economías regionales, que acrecentaron de manera desigual las tasas de desempleo abierto y/o de ocupación informal de las entidades, las cuales provocaron aumentos más que proporcionales en la pobreza alimentaria de las regiones más avanzadas que en las entidades tradicionalmente más rezagadas del país. La prolongación de esa trayectoria durante repetidos trimestres, literalmente acortó las disparidades entre los hogares de las entidades, provocando una clara tendencia a disminuir los contrastes en los niveles interregionales de pobreza alimentaria del país.

Recapitulando: al igual que en la economía estadounidense, y en contraste con la mayor parte de los países latinoamericanos, la gran recesión de 2008-2009 afectó severamente la economía mexicana, acrecentando la TPA de los hogares nacionales. En este proceso, además del encarecimiento de los alimentos básicos, uno de los mecanismos para que la crisis se transmitiera a las condiciones de vida de los hogares fueron, sin duda, los efectos sectorial-regionales de la contracción de la actividad económica sobre el mercado laboral, que acrecentaron de manera diferencial la TDA y/o la TOSI de las entidades federativas.

En efecto, la etapa recesiva del ciclo económico, al originarse en EU, afectó primeramente a las maquiladoras del norte de México y a las manufacturas de exportación del centro del país, acrecentando la TDA en las entidades de 
Gráfica 4. México. Convergencia sigma de las TPA trimestrales estimadas en las 32 entidades federativas y tendencia Hodrick-Prescott, 2005, a 2012

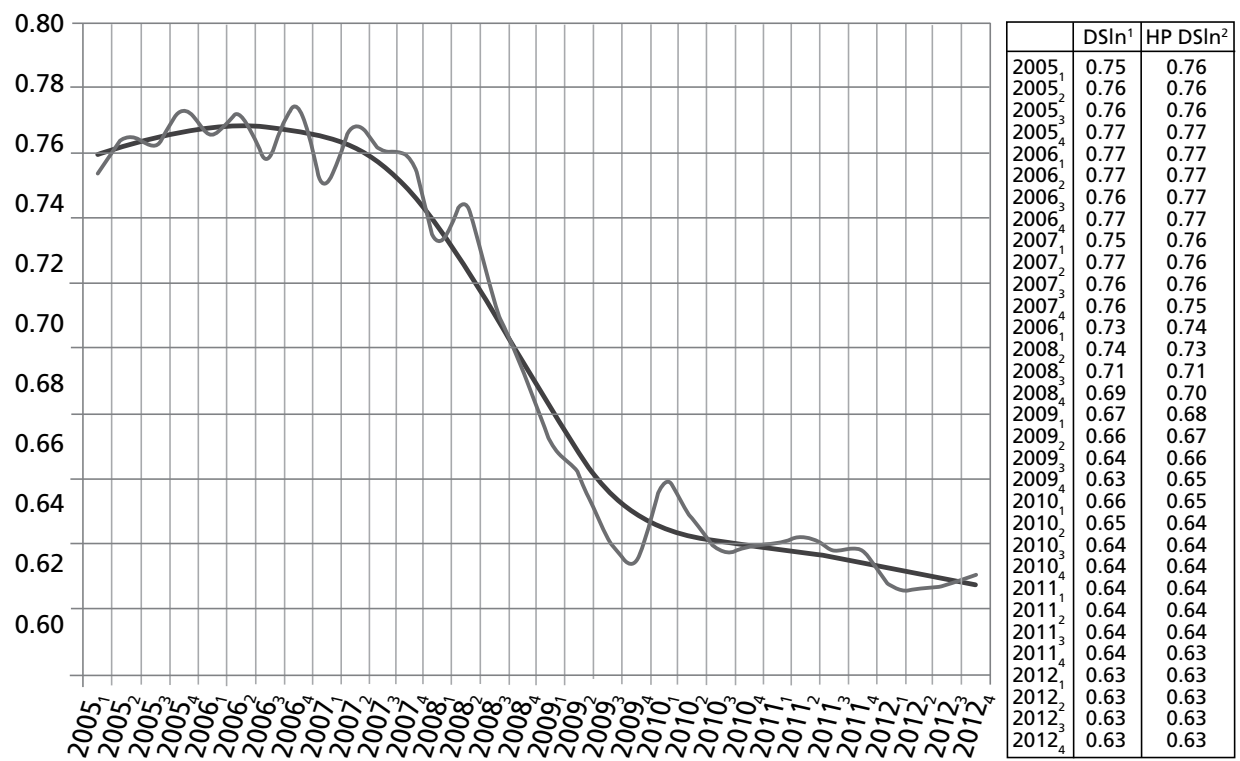

$\longrightarrow \operatorname{DSIn}^{1} \quad$ HP DSIn ${ }^{2}$

${ }^{1}$ Desviación estándar de los logarítmos naturales de las TPA estimadas por trimestre y entidad federativa, con la metodología explicada en el texto.

2 Tendencia calculada con el algoritmo Hodrick-Prescott.

ambas macrorregiones. Al abatirse el empleo y los ingresos, la contracción económica provocó reducciones importantes de las actividades terciarias, tanto comerciales como de servicios, y acrecentó las ocupaciones informales, sobre todo en las entidades del centro y, con algún rezago, también en los estados del sursureste, en algunos de los cuales, sin embargo, había incrementado el desempleo abierto desde el inicio de la fase recesiva, como en los destinos turísticos de Guerrero y Quintana Roo.

Paralelamente, el encarecimiento de los alimentos básicos, tanto nacionales como importados, se sumó a los crecientes niveles de desempleo e informalidad, reduciendo los niveles de bienestar de los hogares mexicanos al incrementar la TDA. Si bien la etapa contractiva sólo duró ocho trimestres, las desfavorables repercusiones sobre el mercado laboral continuaron durante buena parte de la recuperación, que al prolongarse por doce trimestres más, por lo que la TDA siguió aumentando, si bien con menor dinamismo, especialmente en las entidades del centro y del norte, y con menos fuerza en las del sur-sureste. 
Las pruebas estadísticas presentadas dimensionan los efectos del ciclo económico sobre el mercado laboral, el desempleo y la informalidad, así como sus repercusiones en la TPA del país. En este sentido se detecta, de manera estadísticamente significativa, que el impacto cuantitativo del desempleo abierto sobre la pobreza alimentaria es ligeramente menos acusado que el del aumento de la informalidad, que influye de manera directa pero con un rezago trimestral.

Las repercusiones sectorial-regionales del ciclo y sus heterogéneas consecuencias sobre el mercado de trabajo se traducen en una asimetría que incrementó más que proporcionalmente la TPA en las más desarrolladas entidades del norte y el centro del país. Tal efecto fue menor en los estados del sur-sureste, tradicionalmente rezagados, lo que condujo a una trayectoria convergente de las TPA estatales.

Lo anterior pone de relieve, de manera muy clara, las desfavorables secuelas de la recesión en el desempleo y la informalidad, y de ambos en la incidencia de la pobreza alimentaria de los hogares mexicanos. En contraste con los efectos del desempleo, los provocados por el aumento de la informalidad, aunque de intensidad ligeramente mayor, parecieran amortiguar con su rezago trimestral las consecuencias más críticas de la recesión y sus secuelas. Por ello, cobran total pertinencia los propósitos gubernamentales recientemente expuestos de poner en marcha, en un futuro próximo, un programa de aseguramiento universal contra el desempleo, que de llevarse a cabo permitiría prevenir, así sea de manera parcial, que un número creciente de hogares mexicanos quede expuesto a las condiciones más extremas de carencia que se derivan del desempleo.

\section{Anexo}

Cuadro A.1. Resumen de los estadísticos de las variables utilizadas en los ejercicios cuantitativos

\begin{tabular}{|c|c|c|c|c|c|c|c|c|c|}
\hline \multirow{2}{*}{ Variable } & \multirow{2}{*}{ Observaciones } & \multirow{2}{*}{ Media } & \multirow{2}{*}{$\begin{array}{c}\text { Desviación } \\
\text { estándar }\end{array}$} & \multicolumn{2}{|c|}{ Valor } & \multicolumn{2}{|c|}{ Jarque-Bera } & \multicolumn{2}{|c|}{ Raíz unitaria'1 } \\
\hline & & & & Mínimo & Máximo & $\mathrm{JB}$ & $p$ & $t$ & $p$ \\
\hline IGAEP & 1024 & 107.2 & 25.4 & 31.8 & 201.9 & 28.9 & 0.0000 & -31.2497 & 0.0000 \\
\hline IGAES & 1024 & 117.2 & 22.0 & 63.4 & 215.5 & 282.1 & 0.0000 & - 3.3791 & 0.0004 \\
\hline IGAET & 1024 & 121.4 & 10.4 & 99.7 & 161.4 & 125.5 & 0.0000 & -3.4201 & 0.0003 \\
\hline TDA & 1024 & 4.2 & 1.7 & 0.7 & 9.7 & 42.0 & 0.0000 & -2.5200 & 0.0059 \\
\hline TOSI & 1024 & 26.7 & 5.6 & 15.1 & 41.8 & 40.1 & 0.0000 & -3.9117 & 0.0000 \\
\hline TPA & 1024 & 19.0 & 11.0 & 1.2 & 51.1 & 125.1 & 0.0000 & - 3.9097 & 0.0000 \\
\hline
\end{tabular}

1 Prueba de raíz unitaria con información de panel (supone procesos de raíz unitaria común), valor $t$ y nivel de significación de la prueba de Levin, Lin y Chu. 


\section{REFERENCIAS BIBLIOGRÁFICAS}

Abramovitz, Moses (1986), "Catching up, forging ahead and falling behind", Journal of Economic History, 46 (2), pp. 385-406.

Agénor, Pierre R. (2004), "Macroeconomic adjustment and the poor: Analytical issues and cross-country evidence", Journal of Economic Surveys, 18 (3), pp. 351-408.

Aparicio, Ricardo (2009), "La pobreza por ingresos", en Ricardo Aparicio, Verónica Villarespe y Carlos M. Urzúa (coords.), Pobreza en México: Magnitud y perfiles, México, UNAM/IIE/ITESM/CONEVAL, pp. 19-42.

Bane, Mary J., y Ellwood, David T. (1986), "Slipping into and out of poverty", Journal of Human Resources, 21 (1), pp. 1-23.

Bassols, Ángel (1971), La división económica regional de México, México, unAm.

Baulch, Bob, y Hoddinott, John (2000), "Economic mobility and poverty dynamics in developing countries", Journal of Development Studies, 36 (6), pp. 1-24.

BBVA Research (2011), "Situación migración. México", en Fundación BBVA Bancomer. Consultado el 7 de abril de 2014 en: https://www.fundacionbbvabancomer.org/ imagenes/docs/SitMig_2011Nov.pdf.

Benítez, Araceli (2012), "Evolución de la pobreza alimentaria en el ciclo económico de México, un análisis estatal (2005-2010)", tesis de maestría en Economía Social, UAM, México.

Blank, Rebecca M.; Card, David; Levy, Frank, y Meddof, James L. (1993), "Poverty, income distribution and growth: Are still connected?", Brookings Papers on Economic Activity, 1993 (2), pp. 285-339.

Blank, Rebecca M., y Blinder, Alan S. (1985), "Macroeconomics, income distribution, and poverty", NBER Working Papers 1567.

Boeri, Tito, y Ours, Jan van (2008), The economics of imperfect labor markets, New Jersey, Princeton University Press.

Campos, Raymundo; Esquivel, Gerardo, y Lustig, Nora (2012), "The rise and fall of income inequality in Mexico, 1989-2010”, UNU-WIDER Working Paper 2012/10, January.

Campos-Vázquez, Raymundo Miguel (2013), “Efectos de los ingresos no reportados en el nivel y tendencia de la pobreza laboral en México", documento de trabajo IV2013, Centro de Estudios Económicos de El Colegio de México.

Cedlas-unlP y Banco Mundial (2011), "Base de Datos Socioeconómicos de América Latina y el Caribe", en Institut des Hautes Etudes de l'Amérique latine. Consultado el 7 de abril de 2014 en: http://www.iheal.univ-paris3.fr/sites/www.iheal. univ-paris3.fr/files/Pobreza_y_crecimiento_economico_en_AL.pdf 
Chiquiar, Daniel y Ramos-Francia, Manuel (2009), "Competitiveness and growth of the Mexican Economy”, Banco de México, Documentos de Investigación 2009-11, noviembre.

Comité Técnico para la Medición de la Pobreza (2002), Medición de la pobreza. Variantes metodológicas y estimación preliminar, Serie Documentos de Investigación 1, México, SEDESOL.

CONEVAL (2010), Tendencias económicas y sociales de corto plazo y el Índice de la Tendencia Laboral de la Pobreza (ITLP), México, Consejo Nacional de Evaluación de la Política de Desarrollo Social.

- (s.f.a) "Medición de la pobreza. Cuadros ITLP e indicadores de tendencias económicas y sociales al 4to. trimestre de 2013", en CONEVAL. Consultado el 7 de abril de 2014 en: http://www.coneval.gob.mx/Medicion/Paginas/Resultados-anivel-nacional-del-ITLP.aspx.

_ (s.f.b) "Medición de la pobreza. Evolución de las dimensiones de la pobreza 1990-2012", en CONEVAL. Consultado el 7 de abril de 2014 en: http://www. coneval.gob.mx/Medicion/Paginas/Evolucion-de-las-dimensiones-de-lapobreza-1990-2010-.aspx.

_ (s.f.c) "Medición de la pobreza. Mapas de pobreza", en CONEVAL. Consultado el 7 de abril de 2014 en: http://www.coneval.gob.mx/Medicion/Paginas/ Mapas-2005.aspx.

Cortés, Fernando (2010), "Pobreza, desigualdad en la distribución del ingreso y crecimiento económico, 1992-2006", en F. Cortés y O. de Oliveira (coords.), Los grandes problemas de México. V Desigualdad Social, México, El Colegio de México, pp.61-100.

- (2013), "Medio siglo de desigualdad en el ingreso en México", Economía UNAM, 29, mayo-agosto, pp. 12-34.

Cortés, Fernando; Banegas, Israel, y Solís, Patricio (2007), "Pobres con oportunidades", Estudios Sociológicos, 25 (73), pp. 3-40.

Cortés, Fernando; Hernández, Daniel; Hernández Laos, Enrique; Székely, Miguel, y Vera, Hadid (2003), "Evolución y características de la pobreza en México en la última década del siglo xx", Economía Mexicana, nueva época, 12 (2), pp. 295-325.

Cortés, Fernando, y Rubalcava, Rosa M. (1991), Autoexplotación forzada y equidad por empobrecimiento, Jornadas 120, México, El Colegio de México.

Demery, Lionel, y Squire, Lyn (1996), "Macroeconomic adjustment and poverty in Africa: An emerging picture", World Bank Research Observer, 11 (1), February, pp. 39-59. 
Ekes Jr., Alfred E. (2011), The contemporary global economy. A history since 1980, West Sussex, Blackwell Publishing Co.

Esquivel, Gerardo, y Huerta-Pineda, Alejandra (2007), "Las remesas y la pobreza en México: Un enfoque de pareo de puntuación de la propensión”, Integración y Comercio, 27, pp. 47-74.

Fields, Gary S. (1980), "Assessing progress toward greater inequality of income distribution”, en W. P. McGreevy, Third world poverty, Lexington, Mass., Lexington Books, pp. 47-81.

Fundación BBva Bancomer (2013), Anuario de migración y remesas, México.

Janvry, Alain de, y Sadoulet, Elisabeth (1999), "Growth, poverty, and inequality in Latin America: A causal analysis, 1970-94", ponencia a la Conference on Social Protection and Poverty, Inter-American Development Bank, realizada en Washington, DC, el 4 de febrero.

Gerschenkron, Alexander (1962), Economic Backwardness in Historical Perspective, Cambridge, Mass, Harvard University Press.

Griffith-Jones, Stephany, y Ocampo, José A. (2009), "The financial crisis and its impact on developing countries", International Policy Centre for Inclusive Growth, documento de trabajo 53, abril.

Gundersen, Craig, y Ziliak, James P. (2004), "Poverty and macroeconomic performance across space, race and family structure", Demography, 41 (1), pp. 61-86.

Hernández, Gonzalo, y Székely, Miguel (2009), "Labor productivity: the link between economic growth and poverty in Mexico", en M. J. Bane y R. Zenteno (eds.), Poverty and poverty alleviation strategies in North America, Boston, Mass., Harvard University Press, pp. 35-64.

Hernández Laos, Enrique (1992), Crecimiento económico y pobreza en México. Una agenda para la investigación, México, UNAM/CIIH.

(2003), "Distribución del ingreso y pobreza", en E. de la Garza y C. Salas (coords.), La situación del trabajo en México 2003, México:, UAm/Plaza y Valdés, pp. 97-128.

(2006), "Bienestar, pobreza y vulnerabilidad en México: Nuevas estimaciones", Economía UNAM, 9, pp. 14- 32.

- (2009), "Crecimiento, distribución y pobreza (1992-2006)", en R. Aparicio, V. Villarespe y C. M. Urzúa (coords.), Pobreza en México: Magnitud y perfiles, México, UnAM/IIE/ITESM/CONEVAL, pp. 43-98.

Hernández Laos, Enrique; Solís, Ricardo, y Stefanovich, Ana F. (2013), Mercado laboral de profesionistas en México, Documentos, México, ANUIES. 
Hernández Laos, Enrique, y Velázquez, Jorge (2003), Globalización, desigualdad y pobreza. Lecciones de la experiencia mexicana, México, UAM/Plaza y Valdés.

Hoynes, Hillary W. (1999), “The employment, earnings and income of less-skilled workers over the business cycle", Institute for Research on Poverty, discussion paper. 1199-99.

Hoynes, Hilary W.; Page, Marianne E., y Stevens, Ann H. (2006), "Poverty in America", The Journal of Economic Perspectives", 20 (1), pp. 47-68.

ILO (2012), Measuring informality: A statistical manual on the informal sector and informal employment, Geneva, International Labour Office.

INEGI (2012a), Sistema de Cuentas Nacionales de México, información trimestral, base de datos en línea, México.

(2012b), La informalidad laboral. Encuesta Nacional de Ocupación y Empleo. Marco conceptual y metodológico [en línea]. Consultado el 7 de abril de 2014 en: http://www.inegi.org.mx/est/contenidos/Proyectos/encuestas/hogares/ regulares/enoe/doc/Informalidad_marco-met.pdf.

_ (s.f.a) "Encuesta Nacional de Ocupación y Empleo. Consulta interactiva de indicadores estratégicos a partir del 2005 (infoLaboral), en Instituto Nacional de Estadistica y Geografía. Consultado el 7 de abril de 2014 en: http://www3.inegi. org.mx/sistemas/Infoenoe/Default_CONAPO.aspx?s=est\&c=26227 \&p=.

_ (s.f.b), "Encuesta Nacional de Ocupación y Empleo. Consulta interactiva de indicadores estratégicos de 1995 a 2010 (infoLaboral)", en Instituto Nacional de Estadística y Geografía. Consultado el 7 de abril de 2014 en: http://www3.inegi. org.mx/sistemas/infoenoe/default.aspx?s=est\&c=26227\&p=.

_ (s.f.c) "Indicador Global de la Actividad Económica", en Total de las Actividades Económicas (IGAE). Consultado el 7 de abril de 2014 en: http://www.inegi. org.mx/sistemas/bie/?idserPadre=10000200\#D10000200.

Levchenko, Andrei A.; Lewis, Logan T., y Tesar, Linda (2010), "The collapse of international trade during 2008-2009 crisis: In search of the smoking gun”, preliminary version of a paper prepared for the IMF/Banque of France/PSE "Conference on Economic Linkages, Spillovers and the Financial crisis" \& IMF Economic Review.

Loayza, Norman V., y Rigolini, Jamele (2006), "Informality trends and cycles", The World Bank Policy Research Working Paper 4078.

Loayza, Norman V., y Sugawara, Naotaka (2009), "El sector informal en México. Hechos y explicaciones fundamentales", El Trimestre Económico, 76 (4), pp. 887-920. 
Maloney, William F. (1998), “Are labor markets in developing countries dualistic?”, The World Bank Policy Research Working Paper 1941.

(1999), "Does informality imply segmentation in urban labor markets? Evidence from sectoral transitions in Mexico", The World Bank Economic Review, 13 (2), pp. 275-302.

Marshall, Alfred (1977), Principles of Economics, 8a . edición, London, The MacMillan Press, LTD.

Nasar, Sylvia (2011), Grand Pursuit. The story of economic genius, New York, Simon $\&$ Schuster.

OIт (2011), Tendencias mundiales del empleo de 2011. El desafio de la recuperación del empleo [en línea]. Consultado el 7 de abril de 2014 en: http://www.ilo.org/wcmsp5/groups/public/---ed_emp/---emp_elm/---trends/documents/publication/ wcms_150442.pdf

Okun, Arthur M. (1962), "Potential GNP: Its measurement and significance", en ASA, Proceedings of the Business and Economic Statistics Section of the American Statistical Association, Alexandria, American Statistical Association, pp. 89-104.

Perry, Guillermo E.; Maloney, William F.; Arias, Omar S.; Fajnzylber, Pablo; Mason, Andrew D., y Saavedra-Chanduvi, Jaime (2008), Informality, exit and exclusion, Washington, DC, The World Bank.

Pindyck, Robert S., y Rubinfeld, Daniel L. (2001), Econometría: modelos y pronósticos, México, McGraw-Hill.

Presidencia de la República (2012), vI Informe de Gobierno. Anexo Estadístico, México. Ravallion, Martin (2009), "Why don't we see poverty convergence?", Poverty Research Working Paper 4974, The World Bank.

Romer, Christina D., y Romer, David H. (1998), "Monetary policy and the well-being of the poor", documento de trabajo 6793, National Bureau of Economic Research.

Sala-i-Martin, Xavier (2000), Apuntes de crecimiento económico, 2 ${ }^{\mathrm{a}}$. edición, Barcelona, Antoni Bosch editor.

Sayan, Serdar, y Tekin-Koru, Ayca (2008), "Remittances, business cycles and poverty: The recent Turkish experience", товв University of Economics and Technology \& Oregon State University (May).

Seefeldt, Kristin; Abner, Gordon; Bolinger, Joe A.; Xu, Lanlan, y Graham, John D. (2012), "At risk: America's poor during and after the Great Recession", s.l., Indiana University School of Public and Environmental Affairs.

Stevens, Ann H. (1994), "The dynamics of poverty spells: Updating Bane and Ellwood", The American Economic Review, 84 (2), pp. 34-37. 
102 ECONOMÍA: TEORÍA Y PRÁCTICA • Nueva Época, número 40, enero-junio 2014

- (1999), "Climbing out of poverty. Falling back in Measuring the persistence of poverty over multiple spells", The Journal of Human Resources, 34 (3), pp. 557-588.

Stiglitz, Joseph E. (2010), Freefall. America, free markets, and the sinking of the world economy, New York, W. W. Norton \& Co.

Székely, Miguel (2003), Es posible un México con menor pobreza y desigualdad, Documentos de Investigación 5, México, SEDESOL.

Tobin, James (1992), "Poverty in relation to macroeconomic trends, cycles, and policies", Cowles Foundation Discussion Papers 1030R, Cowles Foundation for Research in Economics at Yale University. 